\title{
ON SOME BOUSSINESQ SYSTEMS IN TWO SPACE DIMENSIONS: THEORY AND NUMERICAL ANALYSIS*
}

\author{
Vassilios A. Dougalis ${ }^{1,2}$, Dimitrios E. Mitsotakis ${ }^{1,2}$ and Jean-Claude Saut ${ }^{3}$
}

\begin{abstract}
A three-parameter family of Boussinesq type systems in two space dimensions is considered. These systems approximate the three-dimensional Euler equations, and consist of three nonlinear dispersive wave equations that describe two-way propagation of long surface waves of small amplitude in ideal fluids over a horizontal bottom. For a subset of these systems it is proved that their Cauchy problem is locally well-posed in suitable Sobolev classes. Further, a class of these systems is discretized by Galerkin-finite element methods, and error estimates are proved for the resulting continuous time semidiscretizations. Results of numerical experiments are also presented with the aim of studying properties of line solitary waves and expanding wave solutions of these systems.
\end{abstract}

Mathematics Subject Classification. 35Q53, 65M60, $76 \mathrm{~B} 15$.

Received June 6, 2006. Revised November 26, 2007.

\section{INTRODUCTION}

In this paper we will study Boussinesq systems of the form

$$
\begin{gathered}
\eta_{t}+\nabla \cdot \mathbf{v}+\nabla \cdot \eta \mathbf{v}+a \Delta \nabla \cdot \mathbf{v}-b \Delta \eta_{t}=0 \\
\mathbf{v}_{t}+\nabla \eta+\frac{1}{2} \nabla|\mathbf{v}|^{2}+c \Delta \nabla \eta-d \Delta \mathbf{v}_{t}=0 .
\end{gathered}
$$

These systems have been derived $[6,8]$ as approximations to the three-dimensional Euler equations and describe irrotational free surface flow of an ideal fluid over a horizontal bottom. The independent variable $\mathbf{x}=(x, y)$ represents the position, $t$ is proportional to elapsed time, $\eta=\eta(\mathbf{x}, t)$ is proportional to the deviation of the free surface from its rest position, while $\mathbf{v}=\mathbf{v}(\mathbf{x}, t)=\left(v_{1}(\mathbf{x}, t), v_{2}(\mathbf{x}, t)\right)$ is proportional to the horizontal velocity of the fluid at some height. The coefficients $a, b, c, d$ are given by the formulas

$$
a=\frac{1}{2}\left(\theta^{2}-\frac{1}{3}\right) \nu, b=\frac{1}{2}\left(\theta^{2}-\frac{1}{3}\right)(1-\nu), c=\frac{1}{2}\left(1-\theta^{2}\right) \mu, d=\frac{1}{2}\left(1-\theta^{2}\right)(1-\mu),
$$

Keywords and phrases. Boussinesq systems in two space dimensions, water wave theory, nonlinear dispersive wave equations, Galerkin-finite element methods for Boussinesq systems.

* This research was supported by a Pythagoras grant to the University of Athens, funded by the Greek Ministry of Education and the E. U. European Social Fund.

${ }^{1}$ Department of Mathematics, University of Athens, 15784 Zographou, Greece.

${ }^{2}$ Institute of Applied and Computational Mathematics, F.O.R.T.H., P.O. Box 1.527, 71110 Heraklion, Greece.

${ }^{3}$ UMR de Mathématiques, Université de Paris-Sud, Bâtiment 425, 91405 Orsay, France. jean-claude.saut@math.u-psud.fr

(c) EJP Sciences, SMAI 2007 
where $\nu, \mu$ are real constants and $0 \leq \theta \leq 1$. (The variables in (1.1) are nondimensional but unscaled. If we denote the nondimensional depth variable by $z$, then the bottom of the chamel lies at $z=-1$, while the horizontal velocity of the fluid $\mathbf{v}$ is evaluated at the nondimensional height $z=-1+\theta(1+\eta(\mathbf{x}, t))$.)

As it is explained in detail in [6], the Boussinesq approximation, on which (1.1) is based, is valid when $\varepsilon:=A / h_{0} \ll 1, \lambda / h_{0} \gg 1$, and the Stokes number $S=A \lambda^{2} / h_{0}^{3}$ is of order 1 ; here $A$ is the maximum free elevation above the undisturbed level of the fluid of depth $h_{0}$ and $\lambda$ a typical wavelength. Letting $S=1$ and working in scaled, nondimensional variables, one may derive from the Euler equations, by appropriate expansion in powers of $\varepsilon$, the scaled version of (1.1) in the form

$$
\begin{gathered}
\eta_{t}+\nabla \cdot \mathbf{v}+\varepsilon\left[\nabla \cdot \eta \mathbf{v}+a \Delta \nabla \cdot \mathbf{v}-b \Delta \eta_{t}\right]=\mathcal{O}\left(\varepsilon^{2}\right) \\
\mathbf{v}_{t}+\nabla \eta+\varepsilon\left[\frac{1}{2} \nabla|\mathbf{v}|^{2}+c \Delta \nabla \eta-d \Delta \mathbf{v}_{t}\right]=\mathcal{O}\left(\varepsilon^{2}\right)
\end{gathered}
$$

from which (1.1) follows by rescaling and replacing the right-hand side by 0 .

Concerning the Cauchy problem for (1.1) we shall prove that it is locally well-posed in suitable Sobolev classes, when $b>0$ and $d>0$. This result gives at once the local well-posedness of (1.3) (with zero right-hand side) on a temporal interval $\left[0, T_{0}\right]$ independent of $\varepsilon$. This is not very satisfactory for modelling purposes, since the physically relevant temporal regime for $(1.3)$ is from $O(1 / \varepsilon)$ up to $O\left(1 / \varepsilon^{2}\right)$. In fact, our well-posedness result is shown to be valid for times up to $O\left(1 / \varepsilon^{\alpha}\right)$ for any $a<1 / 2$.

It is worth noticing that the class of fully symmetric systems

$$
\begin{gathered}
\eta_{t}+\nabla \cdot \mathbf{v}+\varepsilon\left[\frac{1}{2} \nabla \cdot \eta \mathbf{v}+a \Delta \nabla \cdot \mathbf{v}-b \Delta \eta_{t}\right]=0 \\
\mathbf{v}_{t}+\nabla \eta+\varepsilon\left[\frac{1}{4} \nabla \eta \eta^{2}+\frac{3}{4}\left(\begin{array}{l}
\partial_{x} v_{1}^{2} \\
\partial_{y} v_{2}^{2}
\end{array}\right)+\frac{1}{4}\left(\begin{array}{l}
\partial_{x} v_{2}^{2} \\
\partial_{y} v_{1}^{2}
\end{array}\right)+\frac{1}{2}\left(\begin{array}{l}
\partial_{y}\left(v_{1} v_{2}\right) \\
\partial_{x}\left(v_{1} v_{2}\right)
\end{array}\right)+c \Delta \nabla \eta-d \Delta \mathbf{v}_{t}\right]=0
\end{gathered}
$$

derived in [8], has an existence theory up to times $O(1 / \varepsilon)$.

On the other hand systems of type (1.1) (or (1.3) with zero right-hand side) are Hamiltonian when $b=d$. However, contrary to the one-dimensional case (see [7]), the conservation of the Hamiltonian camnot be used to yield a global well-posedness result.

Many systems of the form (1.1) possess line solitary wane solutions, as it may be easily seen: We seek travelling wave solutions of (1.1) of the form

$$
\eta(\mathbf{x}, t)=\eta(\xi), \mathbf{v}(\mathbf{x}, t)=\mathbf{v}(\xi),
$$

where $\xi=\boldsymbol{\alpha} \cdot \mathbf{x}-c_{s} t-r_{0}, r_{0} \in \mathbb{R}$, i.e. solutions of (1.1) that travel with constant speed $c_{s}$ without change of form along the direction of the vector $\boldsymbol{\alpha}=\left(\alpha_{x}, \alpha_{y}\right), \alpha_{x}^{2}+\alpha_{y}^{2}=1$. We will assume that $\eta(\xi)$ and $\mathbf{v}(\xi)$ tend to zero, along with their derivatives with respect to $\xi$, as $|\xi| \rightarrow \infty$, and that the component of the velocity of the fluid perpendicular to $\alpha$ is zero.

Making the change of variables $u=\alpha_{x} v_{1}+\alpha_{y} v_{2}, v=\alpha_{y} v_{1}-\alpha_{x} v_{2}$, assuming $v=0$, substituting (1.4) into (1.1) and integrating once, we see that $\eta(\xi)$ and $u(\xi)$ satisfy the equations

$$
\begin{aligned}
-c_{s} \eta+u+\eta u+a u^{\prime \prime}+c_{s} b \eta^{\prime \prime} & =0 \\
-c_{s} u+\eta+\frac{1}{2} u^{2}+c \eta^{\prime \prime}+c_{s} d u^{\prime \prime} & =0
\end{aligned}
$$

for $-\infty<\xi<\infty$, which is precisely the system of o.d.e's satisfied by the solitary wraves of the one-dimensional Boussinesq system of the form (1.1). Existence of the solitary waves for these one-dimensional systems has been studied by Toland [20]: cf. also [11]. For example; in the case of the Bona-Smith family of systems [5], i.e. systems corresponding to the constants

$$
a=0, b=d=\frac{3 \theta^{2}-1}{6}, c=\frac{2-3 \theta^{2}}{3}, \frac{2}{3} \leq \theta^{2} \leq 1,
$$


one may prove, using the techniques of Toland, (cf. $[11,12])$ that solitary waves exist for any $\theta, \theta^{2} \in[2 / 3,1]$, and any value of $c_{s}>1$. Therefore, these systems have line solitary wave solutions for all directions. In case $\frac{7}{9}<\theta^{2}<1$, one may, in addition, find, following the procedure of $[10]$, that for each $\theta ; \theta^{2} \in(7 / 9,1)$ the system (1.1) has one closed form solitary wave solution of the form

$$
\begin{gathered}
\eta_{s}(\mathbf{x}, t)=\eta_{0} \operatorname{sech}^{2}\left(\lambda\left(\boldsymbol{\alpha} \cdot \mathbf{x}-c_{s} t\right)\right) \\
v_{1 s}(\mathbf{x}, t)=B \alpha_{x} \eta_{s}(\mathbf{x}, t) \\
v_{2_{s}}(\mathbf{x}, t)=B a_{y} \eta_{s}(\mathbf{x}, t)
\end{gathered}
$$

where

$$
\begin{array}{cl}
\eta_{0}=\frac{9}{2} \cdot \frac{\theta^{2}-\tau / 9}{1-\theta^{2}}, & \lambda=\frac{1}{2} \sqrt{\frac{3\left(\theta^{2}-\tau / 9\right)}{\left(\theta^{2}-1 / 3\right)\left(\theta^{2}-1 / 3\right)}}, \\
B=\sqrt{\frac{2\left(1-\theta^{2}\right)}{\theta^{2}-1 / 3}}, & c_{s}=\frac{4\left(\theta^{2}-2 / 3\right)}{\sqrt{2\left(1-\theta^{2}\right)\left(\theta^{2}-1 / 3\right)}} .
\end{array}
$$

One may also find cnoidal wave exact solutions following [21], p. 469-470. For example, in the case of the Bona-Smith system with $\theta^{2}=\frac{9}{11}$, we find the exact solution

$$
\eta_{s}(\mathbf{x}, t)=2 \mathrm{cn}^{2}\left[\sqrt{\frac{99}{80}}\left(\boldsymbol{\alpha} \cdot \mathbf{x}-c_{s} t\right)\right], v_{1 s}(\mathbf{x}, t)=B a_{x} \eta_{s}(\mathbf{x}, t), v_{2 s}(\mathbf{x}, t)=B \alpha_{y} \eta_{s}(\mathbf{x}, t),
$$

where, in the notation of $[21], m=\sqrt{2 / 3}, \lambda=2 \sqrt{80 / 99} K(m)$, where $K(m)$ is the complete integral of the first kind.

In what follows, in Section 2 we prove that the Cauchy problem for (1.1) is locally well-posed in suitable Sobolev classes when $b>0$ and $d>0$. This well-posedness result is valid for times up to $O\left(1 / \varepsilon^{\alpha}\right)$ for any $a<1 / 2$. In Section 3 we consider the initial-boundary value problem for the BBM-BBM system (which is the system of Bona-Smith type corresponding to $\theta^{2}=2 / 3$ ) on a bounded convex plane domain with zero Dirichlet boundary conditions. We discretize the problem in space by the standard Galerkin method in subspaces of $H_{0}^{1}$ and prove optimal-order $L^{2}$ and $H^{1}$ error estimates for the resulting semidiscrete approximations of $\eta$ and $\mathbf{v}$. In Section 4 we consider the case $a=0, b=d>0, c<0$, which includes the rest of the Bona-Smith class, i.e. the systems with $2 / 3<\theta^{2} \leq 1$ in (1.7) (for a treatment of an initial boundary-value problem in one dimension, c.f. [4]). We discretize the analogous initial-boundary value problem on rectangles using the standard Galerkin method with tensor products of smooth splines, i.e. subspaces of $H^{2}$, due to the nonvanishing coefficient of the $\Delta \nabla \eta$ term in (1.1). For such semidiscretizations we prove optimal-order error estimates in $H^{2}$ for the approximation of $\eta$ and $H^{1}$ error estimates of sub-optimal order for $\mathbf{v}$. In Section 5 we make an experimental study of the spatial orders of convergence in various norms of fully discrete approximations of the semidiscrete systems presented in Sections 3 and 4. The discretization in time is effected through explicit Runge-Kutta schemes, since the o.d.e. semidiscrete systems are not stiff. We close the paper by reporting on the results of various numerical experiments that we performed, aimed at approximating expanding waves and studying the generation and interactions of line solitary waves for the BBM-BBM and the Bona-Smith system with $\theta^{2}=1$.

\section{WELL-POSEDNESS OF THE BOUSSINESQ SYSTEM (1.1)}

Following the general lines of the analogous proofs of [7] we will prove the following result:

Theorem 2.1. Let $s>0$.

(i) Assume $b>0, d>0, a<0, c<0$ or $b>0, d>0, a=c \geq 0$. Let $\left(\eta_{0}: \mathbf{v}_{0}\right) \in H^{s}\left(\mathbb{R}^{2}\right)^{3}$. Then, there exist $T>0$ and a unique solution

$$
(\eta, \mathbf{v}) \in X_{T}=C\left([0, T] ; H^{s}\left(\mathbb{R}^{2}\right)\right)^{3}
$$

of (1.1) with initial condition $\left(\eta_{0}, \mathbf{v}_{0}\right)$. 
(ii) Assume $b>0, d>0, a=0, c<0$.

Let $\left(\eta_{0}, \mathbf{v}_{0}\right) \in H^{s+1}\left(\mathbb{R}^{2}\right) \times H^{s}\left(\mathbb{R}^{2}\right)^{2}$. Then, there exist $T>0$ and a unique solution

$$
(\eta, \mathbf{v}) \in X_{T}=C\left([0, T] ; H^{s+1}\left(\mathbb{R}^{2}\right)\right) \times C\left([0, T] ; H^{s}\left(\mathbb{R}^{2}\right)\right)^{2}
$$

of (1.1) with initial condition $\left(\eta_{0}, \mathbf{v}_{0}\right)$.

(iii) Assume $b>0, d>0, a<0, c=0$.

Let $\left(\eta_{0}, \mathbf{v}_{0}\right) \in H^{s-1}\left(\mathbb{R}^{2}\right) \times H^{s}\left(\mathbb{R}^{2}\right)^{2}$. Then, there exist $T>0$ and $a$ unique solution

$$
(\eta, \mathbf{v}) \in X_{T}=C\left([0, T] ; H^{s-1}\left(\mathbb{R}^{2}\right)\right) \times C\left([0, T] ; H^{s}\left(\mathbb{R}^{2}\right)\right)^{2}
$$

of (1.1) with initial condition $\left(\eta_{0}, \mathbf{v}_{0}\right)$.

Proof. We write (1.1) in the equivalent form

$$
\begin{gathered}
\eta_{t}+(I-b \Delta)^{-1}[\nabla \cdot \mathbf{v}+\nabla \cdot \eta \mathbf{v}+a \Delta \nabla \cdot \mathbf{v}]=0 \\
\mathbf{v}_{t}+(I-d \Delta)^{-1}\left[\nabla \eta+\frac{1}{2} \nabla|\mathbf{v}|^{2}+c \Delta \nabla \eta\right]=0,
\end{gathered}
$$

and take its Fourier transform:

$$
\frac{\mathrm{d}}{\mathrm{d} t}\left(\begin{array}{c}
\hat{\eta} \\
\hat{v_{1}} \\
\hat{v_{2}}
\end{array}\right)+\mathrm{i}|\mathbf{k}| \mathcal{A}(\mathbf{k})\left(\begin{array}{c}
\hat{\eta} \\
\hat{v_{1}} \\
\hat{v_{2}}
\end{array}\right)+\mathrm{i}\left(\begin{array}{l}
\frac{k_{1} \widehat{\hat{v}}+k_{2} \hat{\eta_{2}}}{1+b|\mathbf{k}|^{2}} \\
\frac{1}{2} \frac{k_{3}}{1+d|\mathbf{k}|^{2}}|\mathbf{v}|^{2} \\
\frac{1}{2} \frac{k_{2}}{1+d|\mathbf{k}|^{2}}|\mathbf{v}|^{2}
\end{array}\right)=0
$$

where $\mathbf{k}=\left(k_{1}, k_{2}\right)$, and

$$
\mathcal{A}(\mathbf{k})=\left(\begin{array}{ccc}
0 & \frac{k_{1}}{|\mathbf{k}|}\left(\frac{1-a|\mathbf{k}|^{2}}{1+b|\mathbf{k}|^{2}}\right) & \frac{k_{2}}{|\mathbf{k}|}\left(\frac{1-a|\mathbf{k}|^{2}}{1+b|\mathbf{k}|^{2}}\right) \\
\frac{k_{1}}{|\mathbf{k}|}\left(\frac{1-c|\mathbf{k}|^{2}}{1+d|\mathbf{k}|^{2}}\right) & 0 & 0 \\
\frac{k_{2}}{|\mathbf{k}|}\left(\frac{1-c|\mathbf{k}|^{2}}{1+d|\mathbf{k}|^{2}}\right) & 0 & 0
\end{array}\right) .
$$

The eigenvalues of $\mathcal{A}(\mathbf{k})$ are $\{0, \pm \sigma(\mathbf{k})\}$, where

$$
\sigma(\mathbf{k})=\left[\frac{\left(1-a|\mathbf{k}|^{2}\right)\left(1-c|\mathbf{k}|^{2}\right)}{\left(1+b|\mathbf{k}|^{2}\right)\left(1+d|\mathbf{k}|^{2}\right)}\right]^{1 / 2} .
$$

Diagonalize the above system:

$$
P^{-1}(\mathbf{k}) \mathcal{A}(\mathbf{k}) P(\mathbf{k})=\left(\begin{array}{ccc}
0 & 0 & 0 \\
0 & \sigma(\mathbf{k}) & 0 \\
0 & 0 & -\sigma(\mathbf{k})
\end{array}\right)
$$

with

where

$$
P(\mathbf{k})=\left(\begin{array}{ccc}
0 & \alpha(\mathbf{k}) & -\alpha(\mathbf{k}) \\
-\frac{k_{2}}{|\mathbf{k}|} & \frac{k_{1}}{|\mathbf{k}|} & \frac{k_{1}}{|\mathbf{k}|} \\
\frac{k_{1}}{|\mathbf{k}|} & \frac{k_{2}}{|\mathbf{k}|} & \frac{k_{2}}{|\mathbf{k}|}
\end{array}\right), P^{-1}(\mathbf{k})=\frac{1}{2 \alpha(\mathbf{k})}\left(\begin{array}{ccc}
0 & -2 \alpha(\mathbf{k}) \frac{k_{2}}{|\mathbf{k}|} & 2 \alpha(\mathbf{k}) \frac{k_{1}}{|\mathbf{k}|} \\
1 & \alpha(\mathbf{k}) \frac{k_{1}}{|\mathbf{k}|} & \alpha(\mathbf{k}) \frac{k_{2}}{|\mathbf{k}|} \\
-1 & \alpha(\mathbf{k}) \frac{k_{1}}{|\mathbf{k}|} & \alpha(\mathbf{k}) \frac{k_{2}}{|\mathbf{k}|}
\end{array}\right),
$$

$$
\alpha(\mathbf{k})=\left[\frac{\left(1+d|\mathbf{k}|^{2}\right)\left(1-a|\mathbf{k}|^{2}\right)}{\left(1-c|\mathbf{k}|^{2}\right)\left(1+b|\mathbf{k}|^{2}\right)}\right]^{1 / 2} .
$$


Performing the change of variables

$$
\left(\begin{array}{l}
\hat{\mu} \\
\hat{\nu}
\end{array}\right)=P^{-1}\left(\begin{array}{l}
\hat{\eta} \\
\hat{\mathbf{v}}
\end{array}\right)
$$

where $\nu=\left(\nu_{1}, \nu_{2}\right)$, we have

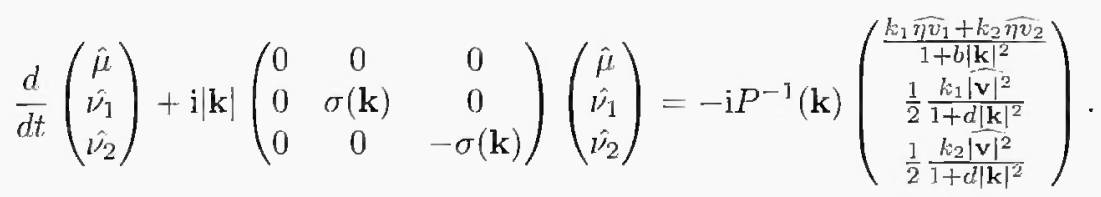

We get

$$
\begin{gathered}
\hat{\mu}(\mathbf{k})=-\frac{k_{2}}{|\mathbf{k}|} \hat{\hat{\jmath}_{1}}(\mathbf{k})+\frac{k_{1}}{|\mathbf{k}|} \hat{v}_{2}(\mathbf{k}) \\
\hat{\nu}_{1}(\mathbf{k})=\frac{1}{2 \alpha(\mathbf{k})} \hat{\eta}(\mathbf{k})+\frac{1}{2} \frac{h_{1}}{|\mathbf{k}|} \hat{v_{1}}(\mathbf{k})+\frac{1}{2} \frac{k_{2}}{|\mathbf{k}|} \hat{v}_{2}(\mathbf{k}) \\
\hat{\nu}_{2}(\mathbf{k})=-\frac{1}{2 \alpha(\mathbf{k})} \hat{\eta}(\mathbf{k})+\frac{1}{2} \frac{k_{1}}{|\mathbf{k}|} \hat{v}_{1}(\mathbf{k})+\frac{1}{2} \frac{k_{2}}{|\mathbf{k}|} \hat{v}_{2}(\mathbf{k}) .
\end{gathered}
$$

Thus, with $H^{s}=H^{s}\left(\mathbb{R}^{2}\right)$, we have

$$
\left(\eta, v_{1}, v_{2}\right) \in H^{s} \times H^{s} \times H^{s} \Rightarrow\left(\mu, \nu_{1}, \nu_{2}\right) \in H^{s} \times H^{s} \times H^{s},
$$

if $\alpha(\mathbf{k})$ is of order 0 (i.e. when $b>0, d>0, a<0, c<0$ or $a=c \geq 0$ ).

On the other hand, when $\alpha(\mathbf{k})$ is of order -1 , i.e. when $b>0, d>0, a=0, c<0$, one has

$$
\left(\eta, v_{1}, v_{2}\right) \in H^{s+1} \times H^{s} \times H^{s} \Rightarrow\left(\mu, \nu, \nu_{2}\right) \in H^{s} \times H^{s} \times H^{s} .
$$

while when $a(\mathbf{k})$ is of order +1 , i.e. when $b>0, d>0, a<0, c=0$,

$$
\left(\eta, v_{1}, v_{2}\right) \in H^{s-1} \times H^{s} \times H^{s} \Rightarrow\left(\mu, \nu_{1}, \nu_{2}\right) \in H^{s} \times H^{s} \times H^{s} .
$$

In the sequel of the proof we give the details in the case that $a(\mathbf{k})$ is of order 0 . The other cases follow analogously. From (2.3)

$$
\frac{\mathrm{d}}{\mathrm{d} t}\left(\begin{array}{c}
\mu \\
\nu_{1} \\
\nu_{2}
\end{array}\right)+\mathcal{B}\left(\begin{array}{c}
\mu \\
\nu_{1} \\
\nu_{2}
\end{array}\right)=\mathcal{F}\left(\begin{array}{c}
\mu \\
\nu_{1} \\
\nu / 2
\end{array}\right)
$$

where $\mathcal{B}$ is the skew-adjoint matrix operator with symbol

$$
\mathrm{i}|\mathbf{k}|\left(\begin{array}{ccc}
0 & 0 & 0 \\
0 & \sigma(\mathbf{k}) & 0 \\
0 & 0 & -\sigma(\mathbf{k})
\end{array}\right) .
$$

The nonlinear tem's Fourier transform is

$$
-\mathbf{i} P^{-1}(\mathbf{k})\left(\begin{array}{c}
\frac{1}{1+b|\mathbf{k}|^{2}}\left(k_{1} \widehat{\eta v_{1}}+k_{2} \widehat{\eta v_{2}}\right) \\
\frac{1}{2} \frac{1}{1+d|\mathbf{k}|^{2}} k_{1} \widehat{|\mathbf{v}|^{2}} \\
\frac{1}{2} \frac{1}{1+d|\mathbf{k}|^{2}} k_{2} \widehat{|\mathbf{v}|^{2}}
\end{array}\right) \text {. }
$$


We note that in terms of $\left(\hat{\mu}, \hat{\nu_{1}}, \hat{\nu_{2}}\right)$,

$$
\left(\begin{array}{c}
\hat{\eta} \\
\hat{v_{1}} \\
\hat{v_{2}}
\end{array}\right)=\left(\begin{array}{c}
\alpha(\mathbf{k}) \hat{\nu_{1}}(\mathbf{k})-\alpha(\mathbf{k}) \hat{\nu_{2}}(\mathbf{k}) \\
-\frac{k_{2}}{\mid \mathbf{k}} \hat{\mu}(\mathbf{k})+\frac{k_{1}}{|\mathbf{k}|} \hat{\nu_{1}}(\mathbf{k})+\frac{k_{1}}{|\mathbf{k}|} \hat{\nu_{2}}(\mathbf{k}) \\
\frac{k_{1} \mid}{|\mathbf{k}|} \hat{\mu}(\mathbf{k})+\frac{k_{2} \mid}{|\mathbf{k}|} \hat{\nu_{1}}(\mathbf{k})+\frac{k_{2}}{|\mathbf{k}|} \hat{\nu_{2}}(\mathbf{k})
\end{array}\right) .
$$

If $\alpha(\mathbf{k})$ is of order 0 , all the pseudo-differential operators involved are of order 0 . So is $P^{-1}(\mathbf{k})$. Now in order to avoid (2.5) of [7], which is no more valid in two dimensions, we use the following lemma, proved by Grisvard [14].

Lemma 2.2 (Grisvard). Let $s_{1}, s_{2}, s_{3} \in \mathbb{R}$ be such that $s_{1} \geq s_{3}, s_{2} \geq s_{3}, s_{1}+s_{2} \geq 0, s_{1}+s_{2}-s_{3}>n / 2$. Then, $(f, g) \mapsto f g$ is bilinear continuous from $H^{s_{1}}\left(\mathbb{R}^{n}\right) \times H^{s_{2}}\left(\mathbb{R}^{n}\right)$ into $H^{s_{3}}\left(\mathbb{R}^{n}\right)$. The result is also valid for a bounded domain $\Omega \subset \mathbb{R}^{r b}$ with Lipschitz boundary.

This lemma implies that, for $s>0$, we have for $n=2$

$$
\left\|\partial_{x_{k}}(I-\Delta)^{-1}(f g)\right\|_{s} \leq\|f g\|_{s-1} \leq C\|f\|_{s}\|g\|_{s}, x_{k}=x, y,
$$

where by $\|\cdot\|_{s}$, we have denoted the norm of $H^{s}\left(\mathbb{R}^{2}\right)$.

The previous considerations show that $\mathcal{F}$ is bilinear continuous from $H^{s} \times H^{s} \times H^{s}$ into itself for $s>0$.

One may also deduce, setting $U=\left(\begin{array}{c}\mu \\ \nu_{1} \\ \nu_{2}\end{array}\right)$, that

$$
U(t)=S(t) U_{0}+\int_{0}^{t} S(t-s) \mathcal{F}(U) \mathrm{d} s,
$$

where $S(t)$ is the unitary group generated by $\mathcal{B}$, defines a contraction in a ball of $X_{T}=C\left([0, T] ; H^{s}\left(\mathbb{R}^{2}\right)\right)^{3}$ for $T$ small enough, if $s>0$, which clearly implies Theorem 2.1 if we transform back to the original variables $\left(\eta, v_{1}, v_{2}\right)$.

Remark 2.3. For modelling purposes it is of interest to consider the scaled version of (1.1), namely

$$
\begin{gathered}
\eta_{t}+\nabla \cdot \mathbf{v}+\varepsilon\left[\nabla \cdot \eta \mathbf{v}+a \Delta \nabla \cdot \mathbf{v}-b \Delta \eta_{t}\right]=0 \\
\mathbf{v}_{t}+\nabla \eta+\varepsilon\left[\frac{1}{2} \nabla|\mathbf{v}|^{2}+c \Delta \nabla \eta-d \Delta \mathbf{v}_{t}\right]=0
\end{gathered}
$$

when $\varepsilon>0$ is small.

One can easily check that Theorem 2.1 provides readily a local well-posedness result for the initial-value problem for (2.5) on a temporal interval $\left[0, T_{0}\right]$, where $T_{0}$ is independent of $\varepsilon$. This is a consequence of the estimate

$$
\left\|\varepsilon \partial_{x_{k}}(I-\varepsilon \Delta)^{-1} f\right\|_{s} \leq\|f\|_{s-1}, x_{k}=x, y, \text { for } 0 \leq \varepsilon \leq 1 .
$$

This result is, of course, not satisfactory. The correct existence time should be at least $O(1 / \varepsilon)$. Note that (2.5) ceases to be formally valid as a model for time scales beyond $O\left(1 / \varepsilon^{2}\right)$. We note that the existence time for the fully symmetric systems (1.4) with $a=c$ and $b, d \geq 0$ was proved to be $O(1 / \varepsilon)[8]$.

We sketch below a proof of the fact that the existence time for $(2.5)$ is at least $O\left(1 / \varepsilon^{1 / 2-}\right)$. For simplicity, we deal only with the $H^{1}$ theory ( $s=1 \mathrm{in}$ Thm. 2.1). The key point is the following lemma.

Lemma 2.4. Let $0 \leq \beta<1$. Then, there exists $C>0$ such that

$$
\left\|\varepsilon \nabla(I-\varepsilon \Delta)^{-1} v\right\|_{1} \leq C \varepsilon^{\beta / 2}\|v\|_{\beta}
$$

for $0<\varepsilon<1$. 
Proof. The proof reduces to proving the following inequality

$$
\frac{\varepsilon|\xi|\left(1+|\xi|^{2}\right)^{1 / 2}}{1+\varepsilon|\xi|^{2}} \leq C \varepsilon^{\beta / 2}\left(1+|\xi|^{2}\right)^{\beta / 2}
$$

i.e.

$$
\frac{\varepsilon|\xi|\left(1+|\xi|^{2}\right)^{(1-\beta) / 2}}{1+\varepsilon|\xi|^{2}} \leq C \varepsilon^{\beta / 2} .
$$

Let $\gamma=\frac{1-\beta}{2} \leq \frac{1}{2}$ and $f_{\varepsilon}=\frac{\varepsilon|\xi|\left(1+|\xi|^{2}\right)^{\gamma}}{1+\varepsilon|\xi|^{2}}$

Setting $X=\varepsilon^{1 / 2}|\xi|$;

$$
f_{\varepsilon}=\frac{\varepsilon^{1 / 2} X\left(1+X^{2} / \varepsilon\right)^{\gamma}}{1+X^{2}}
$$

- When $X^{2} / \varepsilon \leq 1$, we have

$$
f_{\varepsilon} \leq 2^{\gamma} \frac{\varepsilon^{1 / 2} X}{1+X^{2}} \leq 2^{\gamma} \varepsilon
$$

- When $X^{2} / \varepsilon \geq 1$, we have

$$
f_{\varepsilon} \leq 2^{\gamma} \frac{\varepsilon^{1 / 2-\gamma} X^{1+2 \gamma}}{1+X^{2}} \leq 2^{\gamma} \varepsilon^{1 / 2-\gamma}=2^{\frac{1-\beta}{2}} \varepsilon^{\beta / 2} .
$$

Using then Grisvard's Lemma 2.2, we obtain for any $0 \leq \beta<1$

$$
\left\|\varepsilon \nabla(I-\varepsilon \Delta)^{-1}(f g)\right\|_{1} \leq C \varepsilon^{\beta / 2}\|f g\|_{\beta} \leq C \varepsilon^{\beta / 2}\|f\|_{1}\|g\|_{1} .
$$

Note that $\beta$ can be chosen arbitrarily, close (but not equal) to 1 in (2.7).

As a consequence of this lemma, the nonlinear $H^{3}$ estimate in the contraction argument in the proof of Theorem 2.1 will lead to an existence time of order $O\left(\varepsilon^{-\beta / 2}\right)$.

Remark 2.5. As noticed in [7] in the one-dimensional case, (1.1) possesses a Hamiltonian structure when $b=d$. For $n=2$ (1.1) may be written as

$$
\left(\begin{array}{l}
\eta \\
v
\end{array}\right)_{t}+J \operatorname{grad} H(\eta, v)=0
$$

where the skew-adjoint matrix operator $J$ is defined by

$$
J=\left(\begin{array}{cc}
(I-b \Delta)^{-1} & 0 \\
0 & (I-b \Delta)^{-1}
\end{array}\right)\left(\begin{array}{cc}
0 & \text { div } \\
\nabla & 0
\end{array}\right)
$$

and the Hamiltonian $H$ is

$$
H(\eta, \mathbf{v})=\frac{1}{2} \int_{\mathbb{R}^{2}}\left[-c|\nabla \eta|^{2}-a|\nabla \mathbf{v}|^{2}+(1+\eta)|\mathbf{v}|^{2}+\eta^{2}\right]
$$

where $|\nabla \mathbf{v}|^{2}=v_{1, x}^{2}+v_{1, y}^{2}+v_{2, x}^{2}+v_{2, y}^{2}$. It can easily be shown that, when $b=d$, the local solution constructed in Theorem 2.1 preserves the Hamiltonian when $s \geq 1$. Note, however, that contrary to the one-dimensional case, the conservation of $H(\eta, \mathbf{v})$ does not imply a uniform $H^{1}$ bound. 


\section{Galerkin-Finite element semijiscretization of the BBM-BBM system}

In this section we shall analyze the standard Galerkin-finite element semidiscretization of an initial-boundary value problem for the BBM-BBM system, which corresponds to the choice of parameters $a=c=0, b=d=1 / 6$ in (1.1). We shall prove the convergence results for a general $b=d>0$ but will use in the computations $b=d=1 / 6$. (In the sequel we shall use the notation $\mathbf{v}=(u, v)$ for the components of the velocity.) Let $T>0$. We consider the initial-boundary value problem:

$$
\begin{gathered}
\eta_{t}+u_{x}+v_{y}+(\eta u)_{x}+(\eta v)_{y}-b(\Delta \eta)_{t}=0, \\
u_{t}+\eta_{x}+u u_{x}+v v_{x}-b(\Delta u)_{t}=0, \\
v_{t}+\eta_{y}+u u_{y}+v v_{y}-b(\Delta v)_{t}=0, \\
\eta(x, y, 0)=\eta_{0}(x, y), \begin{array}{c}
u(x, y, 0)=u_{0}(x, y), v(x, y, 0)=v_{0}(x, y), \quad(x, y) \in \bar{\Omega} \\
\eta=u=v=0 \text { on } \partial \Omega \times[0, T] .
\end{array} \quad(x, y) \text { on } \Omega, 0<t<T
\end{gathered}
$$

We let $\Omega$ be a convex, plane domain and assume that the problem (3.1) possesses a unique solution $(\eta, u, v)$, which is smooth enough for the purposes of its numerical approximation. (Actually, we have recently proved that problem (3.1) has a unique solution; of. [13].) In the sequel, we let $L^{2}=L^{2}(\Omega)$ with inner product $(\cdot, \cdot)$ and $\|\cdot\|$. We let $H^{k}, k \in \mathbb{Z}^{+}$, denote the usual, $L^{2}$-based Sobolev spaces of functions on $\Omega$, with associated norm $\|\cdot\|_{k}$. We also let $W_{p}^{k}$ the analogous, $L^{p}$ based spaces with norm $\|\cdot\|_{k, p}$. (The norm of $L^{\infty}$ we denote by $\left.\|\cdot\|_{\infty} \cdot\right) C$ will denote a generic constant.

Let $\mathcal{T}_{h}$ denote a regular, quasiuniform triangulation of $\Omega$ with triangles of maximum side $h<1[9]$ and let $S_{h}$ denote a finite-dimensional subspace of $H_{0}^{1}=H_{0}^{1}(\Omega)$, such that for small $h$ and some $r \geq 2$

$$
\inf _{\chi \in S_{h}}\left\{\|v-\chi\|+h\|v-\chi\|_{1}\right\} \leq C h^{s}\|v\|_{s}, \text { for } 1 \leq s \leq r,
$$

when $v \in H^{s} \cap H_{0}^{1}$. Typically, $r-1$ is the degree of the piecewise polynomial functions that usually $S_{h}$ consists of; thus if $S_{h}$ is the space consisting of continuous functions on $\bar{\Omega}$, which are linear in each triangle $\tau$ of $\mathcal{T}_{h}$ and vanish on $\partial \Omega$, then in (3.2) holds with $r=2$.

We define the bilinear form $a_{D}: H_{0}^{1} \times H_{0}^{1} \rightarrow \mathbb{R}$, by the formula

$$
a_{D}(u, v)=(u, v)+b(\nabla u, \nabla v) .
$$

It is obvious, that $a_{D}$ is symmetric, bounded and coercive on $H_{0}^{1} \times H_{0}^{1}$. We also define the elliptic projection $R_{h}: H_{0}^{1} \rightarrow S_{h}$, so that

$$
a_{D}\left(R_{h} v, \chi\right)=a_{D}(v, \chi) \text {, for all } \chi \in S_{h} .
$$

Hence, we have immediately that $\left\|R_{h_{2}} v\right\|_{1} \leq C\|v\|_{1}$, for all $v \in H_{0}^{1}$. It is well-known that, as a consequence of (3.2) and elliptic regularity; we have, for $k=0$ or 1 , that

$$
\left\|v-R_{h} v\right\|_{k} \leq C h^{s-k}\|v\|_{s}, \quad 2 \leq s \leq r ;
$$

for $v \in H^{s} \cap H_{0}^{1}$. Moreover, it is known $(c f .[16,17])$ that

$$
\left\|v-R_{h} v\right\|_{\infty} \leq C(v) \gamma(h), \text { for } v \in W_{\infty}^{r} \cap H_{0}^{1},
$$

where $\gamma(h)=h^{r}|\log h|^{\bar{r}}$ with $\bar{r}=0$ if $r>2$ and $0<\bar{r}<\infty$ if $r=2$.

Last but not least, we mention the inverse estimates for $S_{h}$;

$$
\|\chi\|_{1} \leq C h^{-1}\|\chi\|, \text { and }\|\chi\|_{\infty} \leq C h^{-1}\|\chi\|:
$$

for all $\chi \in S_{h}$, which are consequences of the quasi-uniformity of $\mathcal{T}_{h}$. 
We define now the semidiscrete approximation for the standard Galerkin method for the problem (3.1) as follows. We seek approximations $\eta_{h}, u_{l_{k}}, v_{\eta_{h}}:[0, T] \rightarrow S_{l_{2}}$, such that

$$
\begin{aligned}
& a_{D}\left(\eta_{l t}, \dot{\phi}\right)+\left(u_{h x}, \dot{\phi}\right)+\left(v_{h y}, \dot{\phi}\right)+\left(\left(\eta_{h} u_{h}\right)_{x}, \dot{\phi}\right)+\left(\left(\eta_{h} v_{h}\right)_{y}, \dot{\phi}\right)=0, \quad \dot{\phi} \in S_{h} \\
& a_{D}\left(u_{h t}, \chi\right)+\left(\eta_{h x} ; \chi\right)+\left(u_{h} u_{h x} ; \chi\right)+\left(v_{h} v_{h x} ; \chi\right)=0 ; \quad \chi \in S_{h} \\
& a_{D}\left(v_{h t}, \psi\right)+\left(\eta_{h y}, \psi\right)+\left(u_{h} u_{h y}, \psi\right)+\left(v_{h} v_{h y}, \psi\right)=0, \quad \psi \in S_{h} \\
& \eta_{h}(\cdot, 0)=\eta_{h_{2}}^{0}:=R_{h} \eta_{0}, \quad u_{h}(\cdot, 0)=u_{h}^{0}:=R_{h} u_{0}, \quad v_{h}(\cdot, 0)=v_{h}^{0}:=R_{h} v_{0} .
\end{aligned}
$$

In addition, we define the linear mappings $\hat{f}_{x}, \hat{f}_{y}: L^{2} \rightarrow S_{h}$, which will be useful in the sequel, by the formulas

$$
\begin{aligned}
& a_{D}\left(\hat{f}_{x}(w), \chi\right)=\left(w, \chi_{x}\right), \chi \in S_{h}, \\
& a_{D}\left(\hat{f}_{y}(w), \chi\right)=\left(w, \chi_{y}\right), \chi \in S_{h} .
\end{aligned}
$$

Then, it is clear that (3.6) implies that

$$
\begin{aligned}
& \eta_{h t}=f\left(u_{h}, v_{h}, \eta_{h}\right), \\
& u_{h t}=g\left(u_{h}, v_{h}, \eta_{h}\right), \\
& v_{h t}=z\left(u_{h}, v_{h}, \eta_{h}\right),
\end{aligned}
$$

where

$$
\begin{aligned}
& f\left(u_{h}, v_{h}, \eta_{h}\right):=\hat{f}_{x}\left(u_{h}\right)+\hat{f}_{y}\left(v_{h}\right)+\hat{f}_{x}\left(\eta_{h} u_{h}\right)+\hat{f}_{y}\left(\eta_{h} v_{h}\right), \\
& g\left(u_{h}, v_{h}, \eta_{h}\right):=\hat{f}_{x}\left(\eta_{h}\right)+\frac{1}{2}\left(\hat{f}_{x}\left(u_{h}^{2}\right)+\hat{f}_{x}\left(v_{h}^{2}\right)\right), \\
& \approx\left(u_{h}, v_{h}, \eta_{h}\right):=\hat{f}_{y}\left(\eta_{h}\right)+\frac{1}{2}\left(\hat{f}_{y}\left(u_{h}^{2}\right)+\hat{f}_{y}\left(v_{h}^{2}\right)\right) .
\end{aligned}
$$

We remark that all the above imner products and mappings are well-defined due to Grisvard's Lemma 2.2, which we apply for $n=2, s_{1}=s_{2}=1, s_{3}=0$.

Lemma 3.1. There exists a constant $C$ such that for all $v \in L^{2}$

$$
\left\|\hat{f}_{x}(v)\right\|_{1} \leq C\|v\|, \text { and }\left\|\hat{f}_{y}(v)\right\|_{1} \leq C\|v\| .
$$

Proof. Let $\hat{f}=\hat{f}_{x}$ (the proof for $\hat{f}_{y}$ is analogous). Then, by the coercivity of $a_{D}$ we have

$$
a_{D}(\hat{f}(v), \hat{f}(v)) \geq C\|\hat{f}(v)\|_{1}^{2}
$$

Moreover,

and we have the result.

$$
a_{D}(\hat{f}(v), \hat{f}(v))=\left(v,(\hat{f}(v))_{x}\right) \leq\|v\| \cdot\|\hat{f}(v)\|_{1},
$$

Now, we give the main result of this section.

Proposition 3.2. For small enough $h$, the semidiscrete problem (3.7) possesses a unique solution in $[0, T]$. Also, there exists a constant $C=C(\eta, u, v, T)$, independent of $h$, such that

$$
\left\|\eta-\eta_{h}\right\|+\left\|u-u_{h_{k}}\right\|+\left\|v-v_{h}\right\| \leq C h^{r},
$$

and

for all $t \in[0, T]$.

$$
\left\|\eta-\eta_{h}\right\|_{1}+\left\|u-u_{h}\right\|_{1}+\left\|v-v_{h}\right\|_{1} \leq C h^{r-1},
$$


Proof. We assume that $\|\eta\|_{\infty} \leq M,\|u\|_{\infty} \leq M$ and $\|v\|_{\infty} \leq M$, for $0 \leq t \leq T$. Then, there is a maximal time $t_{l_{k}} \in[0, T]$, such that the solution of the o.d.e. system (3.7) exists and is such that $\left\|\eta_{h}\right\|_{\infty} \leq 2 M,\left\|u_{k}\right\|_{\infty} \leq 2 M$ and $\left\|v_{h}\right\|_{\infty} \leq 2 M$ for all $t \leq t_{h}$. This holds by continuity, because, using (3.5) we have

$$
\left\|u_{h}^{0}\right\|_{\infty} \leq\left\|u_{h_{2}}^{0}-u_{0}\right\|_{\infty}+\left\|u_{0}\right\|_{\infty}=\left\|R_{h_{2}} u_{0}-u_{0}\right\|_{\infty}+\left\|u_{0}\right\|_{\infty} \leq C \gamma(h)+\left\|u_{0}\right\|_{\infty}<2 M,
$$

for $h$ sufficiently small. (Similarly for $\eta_{h}, v_{h}$.) It will be our goal to prove that $t_{h}$ is actually $T$. This, combined with the estimates (3.14) and (3.15) which we will first establish for $t \leq t_{h}$, will allow extending (3.14) and (3.15) up to $t=T$. In the sequel, by " $h$ sufficiently small" we mean that we take $h \leq h_{0}(u, v, \eta, T)$.

Define the quantities

$$
\rho=\eta-R_{h} \eta, \theta=R_{h} \eta-\eta_{h}, \tau=v-R_{h} v, \zeta=R_{h} v-v_{h}, \sigma=u-R_{h} u, \xi=R_{l} u-u_{h},
$$

so that $\theta, \zeta, \xi \in S_{h}$. We also let

$$
e_{\eta}=\eta-\eta_{h}=\rho+\theta, e_{u}=u-u_{h}=\sigma+\xi, e_{v}=v-v_{h}=\tau+\zeta .
$$

Then, by (3.1) and (3.7) we obtain the following relations:

$$
\begin{aligned}
& \theta_{t}=\hat{f}_{x}(\sigma+\xi)+\hat{f}_{y}(\tau+\zeta)+\hat{f}_{x}\left(u \eta-u_{h} \eta_{h}\right)+\hat{f}_{y}\left(v \eta-v_{h} \eta_{h}\right), \\
& \xi_{t}=\hat{f}_{x}(\theta+\rho)+\frac{1}{2}\left\{\hat{f}_{x}\left(u^{2}\right)-\hat{f}_{x}\left(u_{l_{b}}^{2}\right)+\hat{f}_{x}\left(v^{2}\right)-\hat{f}_{x}\left(v_{h}^{2}\right)\right\} \\
& \zeta_{t}=\hat{f}_{y}(\theta+\rho)+\frac{1}{2}\left\{\hat{f}_{y}\left(u^{2}\right)-\hat{f}_{y}\left(u_{h}^{2}\right)+\hat{f}_{y}\left(v^{2}\right)-\hat{f}_{y}\left(v_{h}^{2}\right)\right\} .
\end{aligned}
$$

Because of the fact that

$$
\begin{aligned}
\hat{f}_{x}\left(u^{2}\right)-\hat{f}_{x}\left(u_{h}^{2}\right) & =\hat{f}_{x}\left(u^{2}\right)-\hat{f}_{x}\left((u-\sigma-\xi)^{2}\right) \\
& =\hat{f}_{x}(u(\sigma+\xi))+\hat{f}_{x}\left((\sigma+\xi) u_{h}\right)
\end{aligned}
$$

and that

we obtain by $(3.9)$

$$
\hat{f}_{x}\left(v^{2}\right)-\hat{f}_{x}\left(v_{h}^{2}\right)=\hat{f}_{x}(v(\tau+\zeta))+\hat{f}_{x}\left((\tau+\zeta) v_{h}\right),
$$

$$
\xi_{t}=\hat{f}_{x}(\theta+\rho)+\frac{1}{2}\left\{\hat{f}_{x}(u(\sigma+\xi))+\hat{f}_{x}\left((\sigma+\xi) u_{h}\right)+\hat{f}_{x}(v(\tau+\zeta))+\hat{f}_{x}\left((\tau+\zeta) v_{h}\right)\right\} .
$$

Thus, for all $t \leq t_{h}$ we have

$$
\begin{aligned}
\left\|\xi_{t}\right\|_{1} \leq & \left\|\hat{f}_{x}(\theta+\rho)\right\|_{1}+\frac{1}{2}\left(\left\|\hat{f}_{x}(u(\sigma+\xi))\right\|_{1}\right. \\
& \left.+\left\|\hat{f}_{x}\left((\sigma+\xi) u_{h^{2}}\right)\right\|_{1}+\left\|\hat{f}_{x}(v(\tau+\zeta))\right\|_{1}+\left\|\hat{f}_{x}\left((\tau+\zeta) v_{h}\right)\right\|_{1}\right) \\
\leq & C\left(\|\theta+\rho\|+\|u(\sigma+\xi)\|+\left\|(\sigma+\xi) u_{h}\right\|+\|v(\tau+\zeta)\|+\left\|(\tau+\zeta) v_{h}\right\|\right) \\
\leq & C\left[\|\theta\|+\|\rho\|+\|u\|_{\infty}(\|\sigma\|+\|\xi\|)+\|v\|_{\infty}(\|\tau\|+\|\zeta\|)\right. \\
& \left.+\left\|v_{h}\right\|_{\infty}(\|\tau\|+\|\zeta\|)+\left\|u_{h}\right\|_{\infty}(\|\sigma\|+\|\xi\|)\right]
\end{aligned}
$$

From the last inequality, (3.3), and the remarks in the beginning of the proof we obtain that

$$
\left\|\xi_{t}\right\|_{1} \leq C\left[h^{r}\left(\|\eta\|_{r}+\|u\|_{r}+\|v\|_{r}\right)+\|\theta\|+\|\xi\|+\|\zeta\|\right]
$$


and thus

Similarly, one may prove that

$$
\left\|\xi_{t}\right\|_{1} \leq C\left(h^{r}+\|\theta\|+\|\xi\|+\|\zeta\|\right)
$$

$$
\left\|\zeta_{t}\right\|_{1} \leq C\left(h^{r}+\|\theta\|+\|\xi\|+\|\zeta\|\right) .
$$

Finally, by the fact that

$$
\begin{aligned}
\hat{f}_{x}(u \eta)-\hat{f}_{x}\left(u_{h} \eta_{h}\right) & =\hat{f}_{x}(u \eta)-\hat{f}_{x}((u-\sigma-\xi)(\eta-\rho-\theta)) \\
& =\hat{f}_{x}(u(\rho+\theta))+\hat{f}_{x}\left((\sigma+\xi) \eta_{h}\right)
\end{aligned}
$$

and

we obtain by (3.9)

$$
\hat{f}_{y}(v \eta)-\hat{f}_{y}\left(v_{h} \eta_{h}\right)=\hat{f}_{y}(v(\rho+\theta))+\hat{f}_{y}\left((\tau+\zeta) \eta_{h}\right)
$$

$$
\theta_{t}=\hat{f}_{x}(\sigma+\xi)+\hat{f}_{y}(\tau+\zeta)+\hat{f}_{x}(u(\rho+\theta))+\hat{f}_{x}\left((\sigma+\xi) \eta_{h}\right)+\hat{f}_{y}(v(\rho+\theta))+\hat{f}_{y}\left((\tau+\zeta) \eta_{h}\right)
$$

Thus.

$$
\begin{aligned}
\left\|\theta_{t}\right\|_{1} \leq & C(\|\sigma+\xi\|+\|\tau+\zeta\| \\
& \left.+\|u(\rho+\theta)\|+\left\|(\sigma+\xi) \eta_{h}\right\|+\|v(\rho+\theta)\|+\left\|(\tau+\zeta) \eta_{h}\right\|\right) \\
\leq & C\left(\|\sigma\|+\|\xi\|+\|\tau\|+\|\zeta\|+\|u\|_{\infty}(\|\rho\|+\|\theta\|)+(\|\sigma\|+\|\xi\|)\left\|\eta_{h}\right\|_{\infty}\right. \\
& \left.+\|v\|_{\infty}(\|\rho\|+\|\theta\|)+(\|\tau\|+\|\zeta\|)\left\|\eta_{h}\right\|_{\infty}\right) \\
\leq & C(\|\sigma\|+\|\xi\|+\|\tau\|+\|\zeta\|+\|\rho\|+\|\theta\|),
\end{aligned}
$$

and finally,

From the relations (3.11)-(3.13) we obtain

$$
\left\|\theta_{t}\right\|_{1} \leq C\left(h^{r}+\|\theta\|+\|\xi\|+\|\zeta\|\right) .
$$

$$
\frac{1}{2} \frac{\mathrm{d}}{\mathrm{d} t}\left(\|\theta\|_{1}^{2}+\|\xi\|_{1}^{2}+\|\zeta\|_{1}^{2}\right) \leq\left\|\theta_{t}\right\|_{1} \cdot\|\theta\|_{1}+\left\|\xi_{t}\right\|_{1} \cdot\|\xi\|_{1}+\left\|\zeta_{t}\right\|_{1} \cdot\|\zeta\|_{1} \leq C h^{2 r}+C\left(\|\theta\|_{1}^{2}+\|\xi\|_{1}^{2}+\|\zeta\|_{1}^{2}\right)
$$

and, after using Gronwall's inequality, we get for all $t \leq t /$, the superconvergence result

$$
\|\theta\|_{1}+\|\xi\|_{1}+\|\zeta\|_{1} \leq C h^{r} .
$$

Therefore,

and similarly

$$
\left\|e_{\eta}\right\|+\left\|e_{u}\right\|+\left\|e_{v}\right\| \leq\|\rho\|+\|\theta\|+\|\sigma\|+\|\xi\|+\|\tau\|+\|\zeta\| \leq C h^{r},
$$

$$
\left\|e_{\eta}\right\|_{1}+\left\|e_{u}\right\|_{1}+\left\|e_{v}\right\|_{1} \leq\|\rho\|_{1}+\|\theta\|_{1}+\|\sigma\|_{1}+\|\xi\|_{1}+\|\tau\|_{1}+\|\zeta\|_{1} \leq C h^{T-1} .
$$

Thus, for all $t \leq t_{h}$ we obtain

$$
\left\|\eta-\eta_{h}\right\|+\left\|u-u_{h}\right\|+\left\|v-v_{h}\right\| \leq C h^{r}
$$

and

Hence, for $t \leq t_{h}$

$$
\left\|\eta-\eta_{h}\right\|_{1}+\left\|u-u_{h}\right\|_{1}+\left\|v-v_{h_{h}}\right\|_{1} \leq C h^{r-1}
$$

$$
\begin{aligned}
\left\|u_{h}-u\right\|_{\infty} & \leq\left\|u_{h}-R_{h} u\right\|_{\infty}+\left\|R_{h} u-u\right\|_{\infty} \\
& \leq C h^{-1}\left\|u_{h}-R_{h} u\right\|+C \gamma(h) \\
& \leq C\left(h^{r-1}+\gamma(h)\right) .
\end{aligned}
$$


Hence, $\left\|u_{h}\right\|_{\infty} \leq\left\|u_{h_{h}}-u\right\|_{\infty}+\|u\|_{\infty}<2 M$, for $h$ sufficiently small. (We may argue similarly for $v_{h}, \eta_{h}$.) Therefore, $t_{h}$ is not maximal, and we conclude that $t_{h}$ may be chosen as $T$. The proposition follows from (3.14)-(3.15).

Remark 3.3. The above analysis may be directly adapted to the case of homogeneous Neumann boundary conditions for $\eta$, and homogeneous Dirichlet boundary conditions for $u, v$ on $\partial \Omega$. We obtain the same error estimates. It is also clear that the same error estimates hold if we use tensor products of splines on rectangular domains.

\section{Galerkin semidiscretization of Bona-Smith type systems using tensor PRODUCTS OF SMOOTH SPLINES}

In this section we shall consider the initial-boundary-value problem

$$
\begin{gathered}
\eta_{t}+u_{x}+v_{y}+(\eta u)_{x}+(\eta v)_{y}-b(\Delta \eta)_{t}=0, \\
u_{t}+\eta_{x}+u u_{x}+v v_{x}+c(\Delta \eta)_{x}-b(\Delta u)_{t}=0, \\
v_{t}+\eta_{y}+u u_{y}+v v_{y}+c(\Delta \eta)_{y}-b(\Delta v)_{t}=0, \\
\eta(x, y, 0)=\eta_{0}(x, y), u(x, y, 0)=u_{0}(x, y), v(x, y, 0)=v_{0}(x, y),(x, y) \in \bar{\Omega}, \\
\eta=u=v=0 \text { on } \partial \Omega \times[0, T]
\end{gathered}
$$

where $b>0, c<0$. As we saw, the Bona-Smith family of systems are of the form of $(4.1)$ if $\theta^{2} \in(2 / 3,1]$. We shall let $\Omega$ be a rectangle (for simplicity $\Omega=(0,1) \times(0,1)$ ), and assume that (4.1) possesses a unique solution for $t \in[0, T]$, smooth enough for the purposes of its numerical approximation. We shall discretize (4.1) in space by the standard Galerkin method based on tensor products of smooth splines of order $r \geq 4$, i.e. of degree at least three.

Given a quasiuniform partition of the interval $I=[0,1]$, such that $0=x_{0}<x_{1}<\ldots<x_{N}<x_{N+1}=1$, with $h=\max \left(x_{i+1}-x_{i}\right)$, we define the finite dimensional spaces of splines of order $r \geq 4$,

$$
S p_{h}^{r}(I)=\left\{\phi: \phi \in C^{r-2}[0,1], \phi(0)=\phi(1)=0,\left.\phi\right|_{\left[x_{i}, x_{i+1}\right]} \in \mathbb{P}_{r-1}, 0 \leq i \leq N\right\},
$$

where $\mathbb{P}_{r-1}$ is the space of polynomials of degree $\leq r-1$. Obviously $S p_{b}^{r}$ is a subspace of $H^{r-1}(I) \cap H_{0}^{1}(I)$. We will use the notation $S p_{h}$ instead of $S p_{h}^{r}$ when there is no confusion.

In $\bar{\Omega}=[0,1] \times[0,1]$ consider the quasiuniform partitions in the $x$ - and $y$ - directions, given by $0=x_{0}<x_{1}<$ $\ldots<x_{N}<x_{N+1}=1$ and $0=y_{0}<y_{1}<\ldots<y_{N}<y_{N+1}=1$, respectively, and subdivide $\bar{\Omega}$ by a mesh $\mathcal{T}_{h}$ of rectangles $\left[x_{i}, x_{i+1}\right] \times\left[y_{j}, y_{j+1}\right], 0 \leq i, j \leq N$. Let $h=\max _{i, j}\left(x_{i+1}-x_{i}, y_{j+1}-y_{j}\right)$ and $Q_{h}=S p_{h} \otimes S p_{h}$ consist of all functions $\phi(x, y)$ on $[0,1] \times[0,1]$ of the form

$$
\phi(x, y)=\sum_{i} \sum_{j} c_{i j} \phi_{i}(x) \phi_{j}(y) \equiv \sum_{i, j} c_{i j} \phi_{i j}(x, y),
$$

where $\phi_{i}$ are the basis functions of $S p_{h}$, and $\phi_{i j}(x, y)=\phi_{i}(x) \phi_{j}(y)$, for $1 \leq i, j \leq \operatorname{dim}\left(S p_{h}\right)$, are the corresponding basis functions of $Q_{h}$. It is easily seen that $Q_{h}$ consists of $C^{r-2}$ functions on $\bar{\Omega}$ and is a subspace of $H^{r-1} \cap H_{0}^{1}$. (We shall use the space of bicubic splines, i.e. $Q_{h}$ for $r=4$, for computations.) We list below the approximation properties of $Q_{k}$ that we will need in the sequel. From $[18,19]$ there follow:

$$
\inf _{\lambda \in Q_{h}}\left\{\left\|D^{p}(v-\chi)\right\|\right\} \leq C h^{s-p}\|v\|_{s}, \text { for } 1 \leq s \leq r, \quad 0 \leq p \leq \min (r-1, s), v \in H^{s} \cap H_{0}^{1},
$$

and

$$
\inf _{\chi \in Q_{h}}\left\{\left\|D^{p}(v-\chi)\right\|_{\infty}\right\} \leq C h^{s-p}\|v\|_{s, \infty}, \text { for } 1 \leq s \leq r, 0 \leq p \leq s-1, \text { or } s=p=0, v \in W_{\infty}^{s} \cap H_{0}^{1} .
$$


In addition, we have the following inverse estimates, valid for $\chi \in Q_{h}$,

$$
\|\chi\|_{k, \infty} \leq C h^{-1}\|\chi\|_{k}, \quad k=0,1,\|\chi\|_{k} \leq c h^{-1}\|\chi\|_{k-1}, \quad 0 \leq k \leq r-1 .
$$

We will use again the bilinear form $a_{D}: H_{0}^{1} \times H_{0}^{1} \rightarrow \mathbb{R}$, and the elliptic projection $R_{h_{l}}: H_{0}^{1} \rightarrow Q_{h}$, defined as in the previous section. As a consequence of (4.2) and assuming elliptic regularity, we have

$$
\left\|v-R_{h} v\right\|_{k} \leq C h^{s-k}\|v\|_{s}, \quad 2 \leq s \leq r, \quad k=0,1,2, v \in H^{s} \cap H_{0}^{1} .
$$

We may also assume [17] that

$$
\left\|v-R_{h} v\right\|_{1, \infty} \leq C h^{r-1}\|v\|_{r, \infty} ; \quad v \in W_{\infty}^{r} \cap H_{0}^{1} .
$$

Lemma 4.1. The elliptic projection $R_{l_{3}}$ is stable in $H^{2}$, i.e. $\left\|R_{h} v\right\|_{2} \leq C\|v\|_{2}$ for $v \in H^{2} \cap H_{0}^{1}$.

Proof. First, we recall the fact (elliptic regularity) that if $w \in H^{2} \cap H_{0}^{1}$, then, there exists a constant $C_{1}$ such that

$$
\|w\|_{2} \leq C_{1}\|\Delta w\| .
$$

So we have for $v \in H^{2} \cap H_{0}^{1}$, using the quasiuniformity of the mesh and denoting by $|\cdot|_{s, K}$ the $H^{s}$ seminorm on the element $K$

$$
\begin{aligned}
\left\|R_{h} v\right\|_{2}^{2} & \leq C\left\|\Delta R_{h} v\right\|^{2}=C \sum_{K \in \mathcal{T}_{h}} \int_{K}\left(\Delta R_{h} v\right)^{2}=C \sum_{K \in \mathcal{T}_{h}} \int_{K}\left(\Delta\left(R_{h} v-I_{h} v\right)\right)^{2} \\
& \leq C \sum_{K \in \mathcal{T}_{h}}\left|R_{h} v-I_{h} v\right|_{2, K}^{2} \leq C h^{-2} \sum_{K \in \mathcal{T}_{h}}\left|R_{h} v-I_{h} v\right|_{1, K}^{2}=C h^{-2}\left\|R_{h} v-I_{h} v\right\|_{1}^{2},
\end{aligned}
$$

where $I_{h}$ is the bilinear splines interpolant. It is well known that $\left\|I_{h} v-v\right\|_{1} \leq C h\|v\|_{2}$. Hence

$$
\begin{aligned}
\left\|R_{h} v\right\|_{2} & \leq C h^{-1}\left\|R_{h} v-I_{h} v\right\|_{1} \leq C h^{-1}\left(\left\|R_{h} v-v\right\|_{1}+\left\|v-I_{h} v\right\|_{1}\right) \\
& \leq C h^{-1}\left(\left\|R_{h} v-v\right\|_{1}+C h\|v\|_{2}\right) \leq C\|v\|_{2} .
\end{aligned}
$$

The semidiscrete approximation is now defined as follows. We seek $u_{h}, v_{h}, \eta_{h}:[0, T] \rightarrow Q_{h}$, such that,

$$
\begin{array}{cc}
a_{D}\left(\eta_{h t}, \phi\right)+\left(u_{h x}, \phi\right)+\left(v_{h y}, \phi\right)+\left(\left(\eta_{h} u_{h}\right)_{x}, \phi\right)+\left(\left(\eta_{h} v_{h}\right)_{y}, \phi\right)=0, & \phi \in Q_{h} \\
a_{D}\left(u_{h t}, \chi\right)+\left(\eta_{h x}, \chi\right)+\left(u_{h} u_{h x}, \chi\right)+\left(v_{h} v_{h x}, \chi\right)-c\left(\Delta \eta_{h}, \chi_{x}\right)=0, & \chi \in Q_{h} \\
a_{D}\left(v_{h t}, \psi\right)+\left(\eta_{h y}, \psi\right)+\left(u_{h} u_{h y}, \psi\right)+\left(v_{h} v_{h y}, \psi\right)-c\left(\Delta \eta_{h}, \psi_{y}\right)=0, & \psi \in Q_{h} \\
\eta_{h}(\cdot, 0)=R_{h} \eta_{0}, \quad u_{h}(\cdot, 0)=R_{h} u_{0}, \quad v_{h}(\cdot, 0)=R_{h} v_{0} . &
\end{array}
$$

Furthermore, defining the mappings $\hat{f}_{x}, \hat{f}_{y}: L^{2} \rightarrow Q_{h}$ as in the previous section, we may rewrite (4.7) as

$$
\begin{aligned}
& \eta_{h t}=f\left(u_{h}, v_{h}, \eta_{h}\right), \\
& u_{h t}=g\left(u_{h}, v_{h}, \eta_{h}\right), \\
& v_{h t}=z\left(u_{h}, v_{h}, \eta_{h}\right),
\end{aligned}
$$

where now

$$
\begin{aligned}
f\left(u_{h}, v_{h}, \eta_{h}\right):=\hat{f}_{x}\left(u_{h}\right)+\hat{f}_{y}\left(v_{h}\right)+\hat{f}_{x}\left(\eta_{h} u_{h}\right)+\hat{f}_{y}\left(\eta_{h} v_{h}\right), \\
g\left(u_{h}, v_{h}, \eta_{h}\right):=\hat{f}_{x}\left(\eta_{h}\right)+\frac{1}{2}\left(\hat{f}_{x}\left(u_{h}^{2}\right)+\hat{f}_{x}\left(v_{h}^{2}\right)\right)+c \hat{f}_{x}\left(\Delta \eta_{h}\right), \\
z\left(u_{h}, v_{h}, \eta_{h}\right):=\hat{f}_{y}\left(\eta_{h}\right)+\frac{1}{2}\left(\hat{f}_{y}\left(u_{h}^{2}\right)+\hat{f}_{y}\left(v_{h}^{2}\right)\right)+c \hat{f}_{y}\left(\Delta \eta_{h}\right) .
\end{aligned}
$$


Again, the above relations are well defined by Lemma 2.2 .

Lemma 4.2. (i) There exists a constant $C$ such that for $v \in L^{2}$

$$
\left\|\hat{f}_{x}(v)\right\|_{1} \leq C\|v\|_{\text {: }} \text { and }\left\|\hat{f}_{y}(v)\right\|_{1} \leq C\|v\| .
$$

(ii) There exists a constant $C$ such that for $v \in H^{1}$

$$
\left\|\hat{f}_{x}(v)\right\|_{2} \leq C\|v\|_{1} \text {, and }\left\|\hat{f}_{y}(v)\right\|_{2} \leq C\|v\|_{1} .
$$

Proof. For the proof of (i) cf. Lemma 3.1. Let $v \in H^{1}$. We consider the problem $w-b \Delta w=-v_{x}$, in $\Omega$ with $w=0$ on $\partial \Omega$. Then $\|w\|_{2} \leq C\left\|v_{x}\right\| \leq C\|v\|_{1}$. Thus

$$
a_{D}\left(R_{h} w, \phi\right)=-\left(v_{x}, \dot{\phi}\right)=\left(v, \phi_{x}\right)=a_{D}\left(\hat{f}_{x}(v), \phi\right) \text { for all } \phi \in Q_{h},
$$

i.e. $\hat{f}_{x}(v)=R_{h} w$. Therefore, by Lemma 4.1, $\left\|\hat{f}_{x}(v)\right\|_{2}=\left\|R_{h_{h}} w\right\|_{2} \leq C\|w\|_{2} \leq C\|v\|_{1}$. The proof for $\hat{f}_{y}$ is analogous.

Now, we give the main result of this section.

Proposition 4.3. For small enough h, the semidiscrete problem (4.8) possesses a unique solution in $[0, T]$. There exists a constant $C=C(\eta, u, v, T)$, independent of $h$, such that

$$
\left\|\eta-\eta_{h}\right\|_{2}+\left\|u-u_{l_{l}}\right\|_{1}+\left\|v-v_{l_{b}}\right\|_{1} \leq C h^{r-2},
$$

for all $t \in[0, T]$.

Proof. We assume that $\|\eta\|_{1, \infty} \leq M,\|u\|_{1, \infty} \leq M$ and $\|v\|_{1, \infty} \leq M$ for $0 \leq t \leq T$. Then, for $h$ small enough

$$
\left\|\eta_{h}^{0}\right\|_{1, \infty} \leq\left\|\eta_{h}^{0}-\eta_{0}\right\|_{1, \infty}+\left\|\eta_{0}\right\|_{1, \infty}=\left\|R_{h} \eta_{0}-\eta_{0}\right\|_{1, \infty}+\left\|\eta_{0}\right\|_{1, \infty} \leq C h^{r-1}+\left\|\eta_{0}\right\|_{1, \infty}<2 M .
$$

Similarly we may argue for $u_{h}^{0}$ and $v_{h}^{0}$. Thus, we assume that there is a maximal time $t_{h} \in[0, T]$ such that $\left\|u h_{2}\right\|_{\infty} \leq 2 M,\left\|v_{h}\right\|_{\infty} \leq 2 M$ and $\left\|\eta_{h}\right\|_{1, \infty} \leq 2 M$ for all $t \leq t_{h}$.

We define again

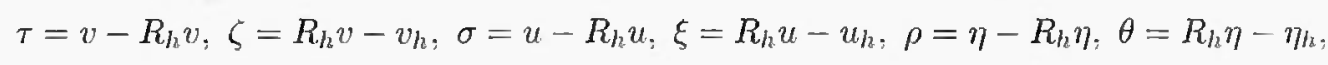

and

$$
e_{u}=u-u_{h}=\sigma+\xi, e_{v}=v-v_{h_{b}}=\tau+\zeta, e_{\eta}=\eta-\eta_{h}=\rho+\theta .
$$

Then, we obtain that

$$
\begin{aligned}
\xi_{t} & =\hat{f}_{x}(\theta+\rho)+\frac{1}{2}\left\{\hat{f}_{x}\left(u^{2}\right)-\hat{f}_{x}\left(u_{h_{h}}^{2}\right)+\hat{f}_{x}\left(v^{2}\right)-\hat{f}_{x}\left(v_{h^{2}}^{2}\right)\right\}+c \hat{f}_{x}(\Delta(\rho+\theta)) \\
\zeta_{t} & =\hat{f}_{y}(\theta+\rho)+\frac{1}{2}\left\{\hat{f}_{y}\left(u^{2}\right)-\hat{f}_{y}\left(u_{h}^{2}\right)+\hat{f}_{y}\left(v^{2}\right)-\hat{f}_{y}\left(v_{h}^{2}\right)\right\}+c \hat{f}_{y}(\Delta(\rho+\theta)) \\
\theta_{t} & =\hat{f}_{x}(\sigma+\xi)+\hat{f}_{y}(\tau+\zeta)+\hat{f}_{x}\left(u \eta-u_{h} \eta_{h}\right)+\hat{f}_{y}\left(v \eta-v_{h} \eta_{h}\right) .
\end{aligned}
$$

Again as in the proof of Proposition 3.2, we obtain using (4.9) that

$$
\xi_{t}=\hat{f}_{x}(\theta+\rho)+\frac{1}{2}\left\{\hat{f}_{x}(u(\sigma+\xi))+\hat{f}_{x:}\left((\sigma+\xi) u_{h}\right)+\hat{f}_{x}(v(\tau+\zeta))+\hat{f}_{x}\left((\tau+\zeta) v_{h}\right)\right\}+c \hat{f}_{x}(\Delta(\rho+\theta)) .
$$


Thus, for all $t \leq t_{h}$ we have

$$
\begin{aligned}
\left\|\xi_{t}\right\|_{1} \leq & C\left(\left\|\hat{f}_{x}(\theta+\rho)\right\|_{1}\left\|\hat{f}_{x}(u(\sigma+\xi))\right\|_{1}\right. \\
& \left.\left.+\left\|\hat{f}_{x}\left((\sigma+\xi) u_{h}\right)\right\|_{1}+\left\|\hat{f}_{x}(v(\tau+\zeta))\right\|_{1}+\left\|\hat{f}_{x}\left((\tau+\zeta) v_{\gamma_{2}}\right)\right\|_{1}\|+\| \hat{f}_{x}(\Delta(\rho+\theta)) \|_{1}\right)\right) \\
\leq & C\left(\|\theta+\rho\|+\|u(\sigma+\xi)\|+\left\|(\sigma+\xi) u_{h_{h}}\right\|+\|v(\tau+\zeta)\|+\left\|(\tau+\zeta) v_{h}\right\|+\|\Delta(\rho+\theta)\|\right) \\
\leq & C\left(\|\theta\|+\|\rho\|+\|u\|_{\infty}(\|\sigma\|+\|\xi\|)+\|v\|_{\infty}(\|\tau\|+\|\zeta\|)+\left\|v_{h}\right\|_{\infty}(\|\tau\|+\|\zeta\|)\right. \\
& \left.+\left\|u_{h}\right\|_{\infty}(\|\sigma\|+\|\xi\|)+\|\rho\|_{2}+\|\theta\|_{2}\right) .
\end{aligned}
$$

From the last inequality we obtain that

$$
\left\|\xi_{t}\right\|_{1} \leq C\left(\left(h^{r-2}\|\eta\|_{r}+h^{T}\|u\|_{r}+h^{T}\|v\|_{r}\right)+\|\theta\|_{2}+\|\xi\|+\|\zeta\|\right),
$$

and thus

Similarly, one may prove that

$$
\left\|\xi_{t}\right\|_{1} \leq C\left(h^{r-2}+\|\theta\|_{2}+\|\xi\|+\|\zeta\|\right) .
$$

$$
\left\|\zeta_{t}\right\|_{1} \leq C\left(h^{r-2}+\|\theta\|_{2}+\|\xi\|+\|\zeta\|\right) .
$$

Finally, as in the proof of Proposition 3.2 we have

$$
\theta_{t}=\hat{f}_{x}(\sigma+\xi)+\hat{f}_{y}(\tau+\zeta)+\hat{f}_{x}(u(\rho+\theta))+\hat{f}_{x}\left((\sigma+\xi) \eta_{h}\right)+\hat{f}_{y}(v(\rho+\theta))+\hat{f}_{y}\left((\tau+\zeta) \eta_{h}\right)
$$

Thus,

$$
\begin{aligned}
\left\|\theta_{t}\right\|_{2} \leq & C\left(\|\sigma+\xi\|_{1}+\|\tau+\zeta\|_{1}\right. \\
& \left.+\|u(\rho+\theta)\|_{1}+\left\|(\sigma+\xi) \eta_{h}\right\|_{1}+\|v(\rho+\theta)\|_{1}+\left\|(\tau+\zeta) \eta_{h}\right\|_{1}\right) \\
\leq & C\left(\|\sigma\|_{1}+\|\xi\|_{1}+\|\tau\|_{1}+\|\zeta\|_{1}+\|u\|_{1, \infty}\left(\|\rho\|_{1}+\|\theta\|_{1}\right)+\left(\|\sigma\|_{1}+\|\xi\|_{1}\right)\left\|\eta_{h}\right\|_{1, \infty}\right. \\
& \left.+\|v\|_{1, \infty}\left(\|\rho\|_{1}+\|\theta\|_{1}\right)+\left(\|\tau\|_{1}+\|\zeta\|_{1}\right)\left\|\eta_{h}\right\|_{1, \infty}\right) \\
\leq & C\left(\|\sigma\|_{1}+\|\xi\|_{1}+\|\tau\|_{1}+\|\zeta\|_{1}+\|\rho\|_{1}+\|\theta\|_{1}\right)
\end{aligned}
$$

and so

$$
\left\|\theta_{t}\right\|_{2} \leq C\left(h^{r-1}+\|\theta\|_{1}+\|\xi\|_{1}+\|\zeta\|_{1}\right) .
$$

From (4.12)-(4.14) we have, using Gronwall's inequality, for all $t \leq t_{h}$

$$
\|\theta\|_{2}+\|\xi\|_{1}+\|\zeta\|_{1} \leq C h^{r-2} .
$$

Hence,

$$
\begin{aligned}
\left\|e_{\eta}\right\|_{2}+\left\|e_{u}\right\|_{1}+\left\|e_{v}\right\|_{1} & \leq\|\rho\|_{2}+\|\theta\|_{2}+\|\sigma\|_{1}+\|\xi\|_{1}+\|\tau\|_{1}+\|\zeta\|_{1} \\
& \leq C h^{T-2}
\end{aligned}
$$

and thus, for all $t \leq t_{h}$ we obtain the result,

$$
\left\|\eta-\eta_{h}\right\|_{2}+\left\|u-u_{h}\right\|_{1}+\left\|v-v_{h}\right\|_{1} \leq C h^{r-2} .
$$

So, for $t \leq t_{h}$, using (4.4) and (4.6)

$$
\begin{aligned}
\left\|\eta_{h}-\eta\right\|_{1, \infty} & \leq\left\|\eta_{h}-R_{h} \eta\right\|_{1, \infty}+\left\|R_{h} \eta-\eta\right\|_{1, \infty} \\
& \leq C h^{-1}\left\|\eta_{h}-R_{h_{h}} \eta\right\|_{1}+C h^{r-1} \\
& \leq C h^{T-3} .
\end{aligned}
$$


Hence, $\left\|\eta_{l}\right\|_{1, \infty} \leq\|\eta\|_{1, \infty}+\left\|\eta_{h}-\eta\right\|_{1, \infty} \leq C h^{r-3}+M<2 M$ for $h$ sufficiently small. Similarly we may argue for $u_{h}, v_{h}$. We may, therefore, conclude that $t_{k}$ can be chosen as $T$. The estimate of the proposition follows from (4.15).

Remark 4.4. The $H^{2}$ estimate for $\eta-\eta_{h}$ in Proposition 4.3 is of optimal order of accuracy, but the $H^{1}$ estimate of the errors of the components of the velocity is suboptimal. In the one-dimensional case, $c f$. [2], one may prove for the analogous initial-boundary-value problem for the Bona-Snith system on a finite interval, the error estimate

$$
\left\|\eta-\eta_{h}\right\|_{1, \infty}+\left\|u-u_{h}\right\|_{\infty} \leq C h^{r-1}
$$

for the standard Galerkin semidiscretization with $C^{2}$ splines of order $r$. Numerical experiments in the onedimensional case with cubic splines indicate that the exponent $r-1=3 \mathrm{in}$ (4.16) is actually sharp.

In the case of periodic boundary conditions at the endpoints of a finite interval, and discretizations with smooth, periodic splines on a uniform mesh, one may prove optimal-order error estimates for $\eta$ and $u$ in the $L^{2}$ norm [2]. These estimates extend easily to discretizations of the initial-periodic-value problem in rectangles using tensor products of smooth periodic splines on uniform meshes.

\section{NumERICAL imPLEMENTATION AND EXPERIMENTAL RATES OF CONVERGENCE}

In this section and in the next one we present the results of numerical experiments that we performed in the case of the BBM-BBM and the Bona-Smith $\left(\theta^{2}=1\right)$ systems using their standard Galerkin spatial discretizations that were analyzed in Sections 3 and 4.

In the case of the BBM-BBM system on rectangles we used a spatial tensor product discretization with continuous, piecewise bilinear elements, a general spatial discretization with triangles, and a tensor product discretization using $C^{2}$, piecewise bicubic elements (bicubic splines) on uniform square meshes. The BonaSmith system was discretized using bicubic splines.

The systems of o.d.e.'s associated with the semidiscretization of the BBM-BBM and the Bona-Smith systems turn out to be nonstiff, cf. [3]. Hence, one may use, for example, explicit Runge-Kutta schemes for their temporal discretization with no restrictions on the time step. In the case of the linear element space discretizations, of second order spatial accuracy, we used the improved Euler method, an explicit Runge-Kutta scheme of secondorder of accuracy; for time stepping; we coupled the bicubic spline spatial discretizations with the matching fourth-order accurate classical explicit Runge-Kutta scheme. Both time marching schemes were used with constant time step $\Delta t$. The error analysis of the fully discrete schemes is long and technical. It has been done in detail in the one-dimensional case, $c f .[2,3]$. We expect that it carries over in two dimensions, yielding for example $L^{2}$ error estimates for the BBM-BBM system of $O\left(h^{r}+k^{p}\right), p=2$ or 4 , and similar $H^{2}-H^{1}$ estimates for the Bona-Smith system.

The attendant linear systems of the tensor product finite element equations at each time step are solved iteratively by the appropriate ITPACK [15] conjugate gradient routine with Jacobi preconditioning. The stopping criterion in this routine is determined by the size of an appropriate relative residual called zeta; which was taken equal to $10^{-6}$ for the second-order and to $10^{-9}$ for the fourth-order schemes. As usual, most of the computational work is due to the quadratures needed to compute the immer products of the right-hand sides of the linear systems. We used (tensor products of) the 3-point, respectively, 5-point, Gaussian quadrature scheme in each spatial interval for the tensor product bilinear, respectively bicubic, discretization. The midpoint (barycenter) rule was used in the case of triangles.

In order to check the spatial convergence rates of the BBM-BBM tensor product codes, we used, in the case of homogeneous Dirichlet boundary conditions for $\eta$ and $\mathbf{v}$, as exact solution on the unit square, the functions

$$
\begin{aligned}
& \eta(x, y, t)=\sin (\pi x)(y-1) y \mathrm{e}^{i} \\
& u(x, y, t)=x \cos ((3 \pi x) / 2) \sin (\pi y) \mathrm{e}^{t} \\
& v(x, y, t)=\sin (\pi x) \cos ((3 \pi y) / 2) \mathrm{e}^{t}
\end{aligned}
$$


TABLE 1. $L^{2}$ errors and convergence rates for the case of Dirichlet boundary conditions. Bilinear elements, BBM-BBM system.

\begin{tabular}{ccccccc}
\hline$N$ & $E_{0}(\eta)$ & $r(\eta)$ & $E_{0}(u)$ & $r(u)$ & $E_{0}(v)$ & $r(v)$ \\
\hline 5 & $3.9656 \mathrm{E}-2$ & - & $6.8208 \mathrm{E}-2$ & - & $6.6550 \mathrm{E}-2$ & - \\
10 & $9.6042 \mathrm{E}-3$ & 2.0457 & $1.6287 \mathrm{E}-2$ & 2.0661 & $1.5825 \mathrm{E}-2$ & 2.0722 \\
15 & $4.2482 \mathrm{E}-3$ & 2.0117 & $7.1836 \mathrm{E}-3$ & 2.0189 & $6.9744 \mathrm{E}-3$ & 2.0207 \\
20 & $2.3855 \mathrm{E}-3$ & 2.0059 & $4.0303 \mathrm{E}-3$ & 2.0090 & $3.9119 \mathrm{E}-3$ & 2.0099 \\
25 & $1.5253 \mathrm{E}-3$ & 2.0039 & $2.5760 \mathrm{E}-3$ & 2.0059 & $2.5003 \mathrm{E}-3$ & 2.0058 \\
30 & $1.0587 \mathrm{E}-3$ & 2.0027 & $1.7879 \mathrm{E}-3$ & 2.0028 & $1.7346 \mathrm{E}-3$ & 2.0053 \\
35 & $7.7735 \mathrm{E}-4$ & 2.0043 & $1.3134 \mathrm{E}-3$ & 2.0008 & $1.2739 \mathrm{E}-3$ & 2.0028 \\
40 & $5.9475 \mathrm{E}-4$ & 2.0051 & $1.0055 \mathrm{E}-3$ & 2.0002 & $9.7511 \mathrm{E}-4$ & 2.0016 \\
\hline
\end{tabular}

TABLE 2. Maximum norm errors and convergence rates for the case of Dirichlet boundary conditions. Bilinear elements, BBM-BBM system.

\begin{tabular}{ccccccc}
\hline$N$ & $E_{\infty}(\eta)$ & $r(\eta)$ & $E_{\infty}(u)$ & $r(u)$ & $E_{\infty}(v)$ & $r(v)$ \\
\hline 5 & $1.3635 \mathrm{E}-1$ & - & $1.0474 \mathrm{E}-1$ & - & $1.0904 \mathrm{E}-1$ & - \\
10 & $3.6638 \mathrm{E}-2$ & 1.8958 & $2.5791 \mathrm{E}-2$ & 2.0219 & $2.6778 \mathrm{E}-2$ & 2.0258 \\
15 & $1.6216 \mathrm{E}-2$ & 2.0101 & $1.1418 \mathrm{E}-2$ & 2.0096 & $1.1849 \mathrm{E}-2$ & 2.0106 \\
20 & $9.0422 \mathrm{E}-3$ & 2.0304 & $6.4137 \mathrm{E}-3$ & 2.0048 & $6.6553 \mathrm{E}-3$ & 2.0053 \\
25 & $5.8256 \mathrm{E}-3$ & 1.9701 & $4.1025 \mathrm{E}-3$ & 2.0024 & $4.2563 \mathrm{E}-3$ & 2.0032 \\
30 & $4.0331 \mathrm{E}-3$ & 2.0168 & $2.8590 \mathrm{E}-3$ & 1.9806 & $2.9551 \mathrm{E}-3$ & 2.0011 \\
35 & $2.9647 \mathrm{E}-3$ & 1.9964 & $2.1069 \mathrm{E}-3$ & 1.9803 & $2.1784 \mathrm{E}-3$ & 1.9781 \\
40 & $2.2704 \mathrm{E}-3$ & 1.9981 & $1.6153 \mathrm{E}-3$ & 1.9895 & $1.6710 \mathrm{E}-3$ & 1.9858 \\
\hline
\end{tabular}

adding an appropriate forcing function. We computed the following error measures at time $T=1$ for each one of the functions $f=\eta$; $u$ or $v$ :

$E_{0}=\left\|f_{h}(\cdot, T)-f(\cdot, T)\right\|, \quad E_{1}=\left\|f_{h}(\cdot, T)-f(\cdot, T)\right\|_{1}, \quad E_{2}=\left\|f_{h}(\cdot, T)-f(\cdot, T)\right\|_{2}, \quad E_{\infty}=\left\|f_{h}(\cdot, T)-f(\cdot, T)\right\|_{\infty}$.

Taking $\Delta t=h$ (we found that the temporal errors did not affect the spatial rates of convergence appreciably in the ranges of $h$ tested), we let the experimental rate of convergence for each of the above error measures $E$ be defined as usual as $\frac{\ln \left(E\left(h_{1}\right) / E\left(h_{2}\right)\right)}{\ln \left(h_{1} / h_{2}\right)}$ for two runs with spatial meshlengths $h_{1}$ and $h_{2}$.

Tables 1-3 show the errors and numerical rates of convergence $r(f)$ for $f=\eta, u$ or $v$ for the BBM-BBM system discretized in space by bilinear elements, that correspond to $r=2$ in the estimates of Section 3 . The first column $N=1 / h$ represents the number of spatial intervals used in each direction $x, y$. As expected from Proposition 3.2, the $L^{2}$ and $H^{1}$ rates turn out to be equal to 2 and 1 , respectively. The maximum norm convergence rates are also practically equal to 2. The same rates are obtained in the case of homogeneous Neumam boundary conditions for $\eta$ and $\mathrm{v}$. The analogous rates in the bicubic spline discretization case were found to be, as expected, 4,3 , and 4 in the $L^{2}, H^{1}$ and $L^{\infty}$ norms, respectively.

We turn now to the Bona-Smith system with $\theta^{2}=1$. We considered homogeneous Dirichlet boundary conditions for $\eta$ and $\mathbf{v}$ on the unit square and used as exact solution (with an appropriate right-hand side) the functions

$$
\begin{aligned}
& \eta(x, y, t)=\mathrm{e}^{(2 t+(x-2)(y-2))}(x-1) x^{2}(y-1) y \\
& u(x, y, t)=\mathrm{e}^{(2 t+x y)}(x-1) x(y-1) y^{2} \\
& v(x, y, t)=\mathrm{e}^{(2 t+(x-1)(1-y))}(x-1) x^{2}(y-1) y
\end{aligned}
$$


TABLE 3. $H^{1}$ errors and convergence rates for the case of Dirichlet boundary conditions. Bilinear elements, BBM-BBM system.

\begin{tabular}{ccccccc}
\hline$N$ & $E_{1}(\eta)$ & $r(\eta)$ & $E_{1}(u)$ & $r(u)$ & $E_{1}(v)$ & $r(v)$ \\
\hline 5 & $3.8318 \mathrm{E}-1$ & & 1.4128 & & 1.4135 & - \\
10 & $1.6079 \mathrm{E}-1$ & 1.2527 & $7.0024 \mathrm{E}-1$ & 1.0127 & $7.0034 \mathrm{E}-1$ & 1.0131 \\
15 & $1.0303 \mathrm{E}-1$ & 1.0977 & $4.6611 \mathrm{E}-1$ & 1.0037 & $4.6614 \mathrm{E}-1$ & 1.0039 \\
20 & $7.6151 \mathrm{E}-2$ & 1.0508 & $3.4939 \mathrm{E}-1$ & 1.0018 & $3.4941 \mathrm{E}-1$ & 1.0019 \\
25 & $6.0501 \mathrm{E}-2$ & 1.0309 & $2.7945 \mathrm{E}-1$ & 1.0010 & $2.7945 \mathrm{E}-1$ & 1.0011 \\
30 & $5.0226 \mathrm{E}-2$ & 1.0208 & $2.3284 \mathrm{E}-1$ & 1.0007 & $2.3284 \mathrm{E}-1$ & 1.0007 \\
35 & $4.2952 \mathrm{E}-2$ & 1.0149 & $1.9956 \mathrm{E}-1$ & 1.0005 & $1.9956 \mathrm{E}-1$ & 1.0005 \\
40 & $3.7527 \mathrm{E}-2$ & 1.0112 & $1.7461 \mathrm{E}-1$ & 1.0003 & $1.7461 \mathrm{E}-1$ & 1.0004 \\
\hline
\end{tabular}

TABLE 4. $L^{2}$ errors and convergence rates for the case of Dirichlet boundary conditions. Bicubic elements, Bona-Smith system, $\theta^{2}=1$.

\begin{tabular}{ccccccc}
\hline$N$ & $E_{0}(\eta)$ & $r(\eta)$ & $E_{0}(u)$ & $r(u)$ & $E_{0}(v)$ & $r(v)$ \\
\hline 10 & $1.9312 \mathrm{E}-4$ & - & $2.9515 \mathrm{E}-3$ & - & $0.6795 \mathrm{E}-4$ & - \\
20 & $2.3437 \mathrm{E}-5$ & 3.0426 & $3.6199 \mathrm{E}-4$ & 3.0274 & $8.3433 \mathrm{E}-5$ & 3.0257 \\
30 & $6.8919 \mathrm{E}-6$ & 3.0187 & $1.0630 \mathrm{E}-4$ & 3.0220 & $2.4556 \mathrm{E}-5$ & 3.0164 \\
40 & $2.8981 \mathrm{E}-6$ & 3.0112 & $4.4623 \mathrm{E}-5$ & 3.0173 & $1.0324 \mathrm{E}-5$ & 3.0120 \\
50 & $1.4812 \mathrm{E}-6$ & 3.0076 & $2.2775 \mathrm{E}-5$ & 3.0141 & $5.2746 \mathrm{E}-6$ & 3.0095 \\
60 & $8.5632 \mathrm{E}-7$ & 3.0058 & $1.3151 \mathrm{E}-5$ & 3.0119 & $3.0481 \mathrm{E}-6$ & 3.0077 \\
70 & $5.3889 \mathrm{E}-7$ & 3.0043 & $8.2687 \mathrm{E}-6$ & 3.0103 & $1.9174 \mathrm{E}-6$ & 3.0069 \\
80 & $3.6081 \mathrm{E}-7$ & 3.0043 & $5.5326 \mathrm{E}-6$ & 3.0091 & $1.2836 \mathrm{E}-6$ & 3.0055 \\
\hline
\end{tabular}

TABLE 5. Maximum errors and convergence rates for the case of Dirichlet boundary conditions. Bicubic elements, Bona-Smith system, $\theta^{2}=1$.

\begin{tabular}{ccccccc}
\hline$N$ & $E_{\infty}(\eta)$ & $r(\eta)$ & $E_{\infty}(u)$ & $r(u)$ & $E_{\infty}(v)$ & $r(v)$ \\
\hline 10 & $5.8697 \mathrm{E}-4$ & - & $6.9955 \mathrm{E}-3$ & - & $1.5904 \mathrm{E}-3$ & - \\
20 & $4.6892 \mathrm{E}-5$ & 3.6458 & $1.1379 \mathrm{E}-3$ & 2.6200 & $2.5654 \mathrm{E}-4$ & 2.6321 \\
30 & $1.3626 \mathrm{E}-5$ & 3.0479 & $3.6926 \mathrm{E}-4$ & 2.7757 & $8.2633 \mathrm{E}-5$ & 2.7940 \\
40 & $5.7008 \mathrm{E}-6$ & 3.0290 & $1.6572 \mathrm{E}-4$ & 2.7849 & $3.6999 \mathrm{E}-5$ & 2.7930 \\
50 & $2.9065 \mathrm{E}-6$ & 3.0188 & $8.8176 \mathrm{E}-5$ & 2.8276 & $1.9659 \mathrm{E}-5$ & 2.8336 \\
60 & $1.6769 \mathrm{E}-6$ & 3.0166 & $5.2286 \mathrm{E}-5$ & 2.8663 & $1.1670 \mathrm{E}-5$ & 2.8603 \\
70 & $1.0540 \mathrm{E}-6$ & 3.0122 & $3.3525 \mathrm{E}-5$ & 2.8830 & $7.4883 \mathrm{E}-6$ & 2.8785 \\
80 & $7.0495 \mathrm{E}-7$ & 3.0123 & $2.2756 \mathrm{E}-5$ & 2.9017 & $5.0889 \mathrm{E}-6$ & 2.8928 \\
\hline
\end{tabular}

We discretized this problem with bicubic splines coupled with the classical, explicit, fourth-order Runge-Kutta scheme for time stepping. Table 4 shows that the $L^{2}$ rates of convergence for all three variables are, apparently, equal to 3. The maximum norm rates (Tab. 5) are found to be approximately equal to 3 for $\eta$, $u$, and $v$. The $H^{1}$ rates (Tab. 6) seem to be equal to 3 for $\eta$ and very close to 2.5 for the two velocity components. Table 7 shows the $H^{2}$ errors and rates; the latter are equal to 2 for $\eta$ (as proved in Prop. 4.3), and practically equal to 1.5 for $u$ and $v$.

Our conclusions from these numerical experiments in the case of the Bona-Smith system are, first, that the optimal $H^{2}$ rate for $\eta$ ( $r=2$ in the case of cubic splines) is, of course, confirmed. The $H^{1}$ rates for $u$ and $v$ are found to be slightly better than the rates (equal to 2) predicted by Proposition 4.3. The $L^{2}$ rates seem 
TABLE 6. $H^{1}$ errors and convergence rates for the case of Dirichlet boundary conditions. Bicubic elements, Bona-Smith system, $\theta^{2}=1$.

\begin{tabular}{ccccccc}
\hline$N$ & $E_{1}(\eta)$ & $r(\eta)$ & $E_{1}(u)$ & $r(u)$ & $E_{1}(v)$ & $r(v)$ \\
\hline 10 & $4.4529 \mathrm{E}-3$ & - & $6.1226 \mathrm{E}-2$ & - & $1.3824 \mathrm{E}-2$ & - \\
20 & $5.8385 \mathrm{E}-4$ & 2.9310 & $1.0857 \mathrm{E}-2$ & 2.4954 & $2.4703 \mathrm{E}-3$ & 2.4844 \\
30 & $1.7626 \mathrm{E}-4$ & 2.9538 & $3.9295 \mathrm{E}-3$ & 2.5065 & $8.9999 \mathrm{E}-4$ & 2.4903 \\
40 & $7.5093 \mathrm{E}-5$ & 2.9659 & $1.9102 \mathrm{E}-3$ & 2.5073 & $4.3930 \mathrm{E}-4$ & 2.4929 \\
50 & $3.8678 \mathrm{E}-5$ & 2.9731 & $1.0918 \mathrm{E}-3$ & 2.5068 & $2.5178 \mathrm{E}-4$ & 2.4945 \\
60 & $2.2474 \mathrm{E}-5$ & 2.9778 & $6.9136 \mathrm{E}-4$ & 2.5061 & $1.5974 \mathrm{E}-4$ & 2.4953 \\
70 & $1.4194 \mathrm{E}-5$ & 2.9811 & $4.6985 \mathrm{E}-4$ & 2.5056 & $1.0871 \mathrm{E}-4$ & 2.4964 \\
80 & $9.5298 \mathrm{E}-6$ & 2.9835 & $3.3626 \mathrm{E}-4$ & 2.5051 & $7.7900 \mathrm{E}-5$ & 2.4963 \\
\hline
\end{tabular}

TABLE 7. $H^{2}$ errors and convergence rates for the case of Dirichlet boundary conditions. Bicubic elements, Bona-Smith system, $\theta^{2}=1$.

\begin{tabular}{ccccccc}
\hline$N$ & $E_{2}(\eta)$ & $r(\eta)$ & $E_{2}(u)$ & $r(u)$ & $E_{2}(v)$ & $r(v)$ \\
\hline 10 & $3.2970 \mathrm{E}-1$ & - & $2.5455 \mathrm{E}-0$ & - & $5.6973 \mathrm{E}-1$ & - \\
20 & $8.0418 \mathrm{E}-2$ & 2.0355 & $8.8190 \mathrm{E}-1$ & 1.5292 & $1.9984 \mathrm{E}-1$ & 1.5113 \\
30 & $3.5486 \mathrm{E}-2$ & 2.0176 & $4.7481 \mathrm{E}-1$ & 1.5270 & $1.0847 \mathrm{E}-1$ & 1.5069 \\
40 & $1.9896 \mathrm{E}-2$ & 2.0113 & $3.0644 \mathrm{E}-1$ & 1.5220 & $7.0349 \mathrm{E}-2$ & 1.5052 \\
50 & $1.2710 \mathrm{E}-2$ & 2.0082 & $2.1837 \mathrm{E}-1$ & 1.5184 & $5.0290 \mathrm{E}-2$ & 1.5042 \\
60 & $8.8163 \mathrm{E}-3$ & 2.0063 & $1.6564 \mathrm{E}-1$ & 1.5156 & $3.8232 \mathrm{E}-2$ & 1.5034 \\
70 & $6.4721 \mathrm{E}-3$ & 2.0052 & $1.3117 \mathrm{E}-1$ & 1.5136 & $3.0324 \mathrm{E}-2$ & 1.5034 \\
80 & $4.9522 \mathrm{E}-3$ & 2.0043 & $1.0719 \mathrm{E}-1$ & 1.5121 & $2.4811 \mathrm{E}-2$ & 1.5024 \\
\hline
\end{tabular}

to be suboptimal, i.e. equal to 3 for all three variables: this is expected from the completely analogous onedimensional results [2]. The maximum norm rates seem to be approach 3 for all three variables, exactly as in the one-dimensional case. The $H^{1}$ estimate for $\eta$ seems to be of optimal rate (three) and slightly suboptimal for $u$ and $v$.

It is worthwhile to note that numerical experiments in the case of the Bona-Smith system with periodic boundary conditions in $x$ and $y$ on the unit square, using tensor products of periodic cubic splines on a uniform mesh, yielded the optimal $L^{2}$ rates (equal to 4 ) for all three variables. This is, of course, expected from the analogous theoretical results in the periodic; one-dimensional case, [2], that are preserved by the tensor product spaces.

We also discretized the BBM-BBM system with continuous, piecewise linear elements on a regular triangular mesh. For the temporal integration of the systems of o.d.e.'s associated with this semidiscretization we used, again, the improved Euler method. The attendant linear systems of the finite elements equations at each time step are solved iteratively by the appropriate ITPACK SOR, routine, where the relative residual was taken to be equal to $10^{-7}$.

We checked spatial convergence rates of this code in the case of homogeneous Neumann boundary conditions for $\eta$ and homogeneous Dirichlet boundary conditions for $u$ and $v$, using a triangular mesh on the unit square, consisting of isosceles orthogonal triangles, with perpendicular sides of length $h=\sqrt{2 / N}$, where $N$ is the number of triangles we used. We also used as exact solution (with an appropriate right-hand side) the functions

$$
\begin{aligned}
& \eta(x, y, t)=\cos (\pi x) \cos (\pi y) \mathrm{e}^{t} \\
& u(x, y, t)=x \cos ((\pi x) / 2) \sin (\pi y) \mathrm{e}^{t} \\
& v(x, y, t)=y \cos ((\pi y) / 2) \sin (\pi x) \mathrm{e}^{t}
\end{aligned}
$$


TABLE 8, $L^{2}$ errors and convergence rates for the case of mixed boundary conditions. Linear elements on triangular mesh, BBM-BBM system.

\begin{tabular}{ccccccc}
\hline$N$ & $E_{0}(\eta)$ & $r(\eta)$ & $E_{0}(u)$ & $r(u)$ & $E_{0}(v)$ & $r(v)$ \\
\hline 128 & $1.1546 \mathrm{E}-1$ & - & $9.0589 \mathrm{E}-2$ & - & $9.0589 \mathrm{E}-2$ & - \\
512 & $2.4976 \mathrm{E}-2$ & 2.2088 & $1.7572 \mathrm{E}-2$ & 2.3660 & $1.7572 \mathrm{E}-2$ & 2.3660 \\
2048 & $5.4633 \mathrm{E}-3$ & 2.1927 & $3.5819 \mathrm{E}-3$ & 2.2945 & $3.5819 \mathrm{E}-3$ & 2.2945 \\
8192 & $1.2666 \mathrm{E}-3$ & 2.1087 & $7.9123 \mathrm{E}-4$ & 2.1785 & $7.9123 \mathrm{E}-4$ & 2.1785 \\
32768 & $3.1122 \mathrm{E}-4$ & 2.0250 & $1.8654 \mathrm{E}-4$ & 2.0846 & $1.8654 \mathrm{E}-4$ & 2.0846 \\
\hline
\end{tabular}

TABLE 9. $H^{1}$ errors and convergence rates for the case of mixed boundary conditions. Linear elements on triangular mesh. BBM-BBM system.

\begin{tabular}{ccccccc}
\hline$N$ & $E_{1}(\eta)$ & $r(\eta)$ & $E_{1}(u)$ & $r(u)$ & $E_{1}(v)$ & $r(v)$ \\
\hline 128 & $9.6646 \mathrm{E}-1$ & - & $6.3426 \mathrm{E}-1$ & - & $6.3426 \mathrm{E}-1$ & - \\
512 & $4.4960 \mathrm{E}-1$ & 1.1040 & $2.1906 \mathrm{E}-1$ & 1.5337 & $2.1906 \mathrm{E}-1$ & 1.5337 \\
2048 & $2.2146 \mathrm{E}-1$ & 1.0215 & $9.1678 \mathrm{E}-2$ & 1.2567 & $9.1678 \mathrm{E}-2$ & 1.2567 \\
8192 & $1.1050 \mathrm{E}-1$ & 1.0030 & $4.2849 \mathrm{E}-2$ & 1.0973 & $4.2849 \mathrm{E}-2$ & 1.0973 \\
32768 & $5.5235 \mathrm{E}-2$ & 1.0003 & $2.0857 \mathrm{E}-2$ & 1.0386 & $2.0857 \mathrm{E}-2$ & 1.0386 \\
\hline
\end{tabular}
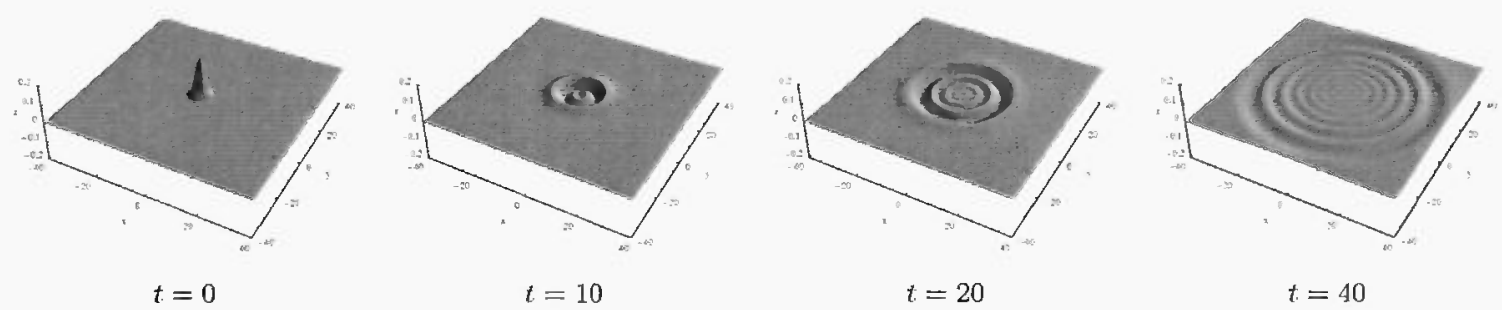

Figure 1. $\eta(x, y, t)$ at $t=0,10,20,40$, BBM-BBM, zero Neumann b. c.

Tables 8-9 show that the $L^{2}$ and $H^{1}$ rates of convergence seem to be of optimal order, i.e. approximately equal to 2 and 1, respectively.

\section{NUMERICAL EXPERIMENTS}

In this section, we present the results of some numerical experiments that we have performed with the fully discrete BBM-BBM and Bona-Smith $\left(\theta^{2}=1\right)$ codes described in the previous section. Tensor product elements were used in numerical experiments 6.1-6.5, and triangular elements in 6.6 and 6.7. The labels RK2, RK4 refer to the time-stepping procedures with the explicit Runge-Kutta schemes of accuracy 2,4 , respectively.

\subsection{Expanding symmetric waves}

In Figures 1-8 we present the results of numerical simulations of the evolution of initially localized heaps of fluid of initial velocity zero. All computations were performed on the square $\Omega=(-40,40) \times(-40,40)$.

In Figure 1 we present the evolution of the $\eta$ profile emanating from the radially symmetric initial data $\eta_{0}(x, y)=0.2 \exp \left(-\left(x^{2}+y^{2}\right) / 5\right), u_{0}(x, y)=v_{0}(x, y)=0$, under the BBM-BBM system with zero Neumann boundary conditions for $\eta ; u$ and $v$ on $\partial \Omega$. (Bilinear elements-RK2, square mesh, $h=0.5, \Delta t=0.05$.) Observe 

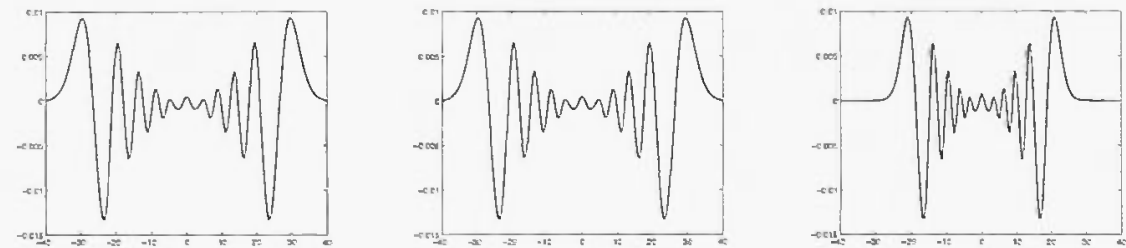

Figure 2. Cross sections in the $(0,1),(1,0)$ and $(1,1)$ directions of $\eta(2, y, t)$ at $t=30$. Evolution of Figure 1.

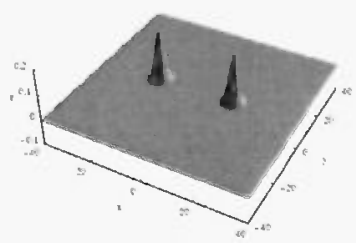

$t=0$

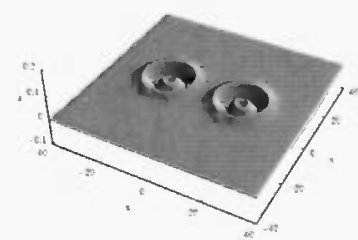

$t=10$

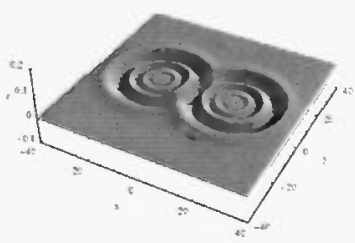

$1=20$

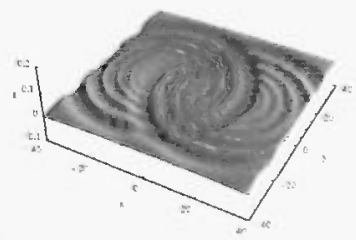

$t=40$

Figure 3. $\eta(x, y, t)$ at $t=0,10,20,40$, BBM-BBM, zero Neumann b. c.

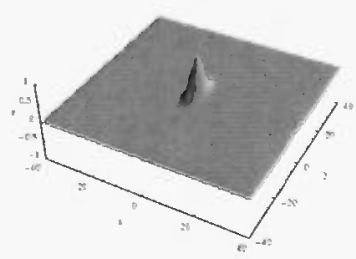

$t=0$

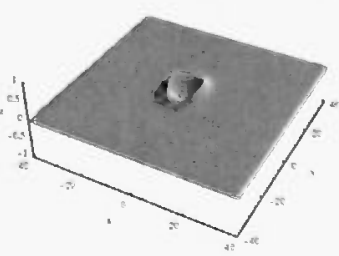

$t=5$

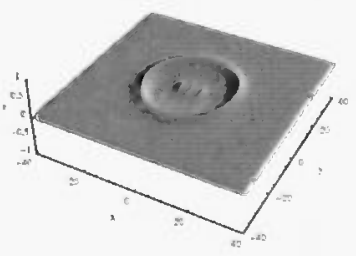

$t=15$

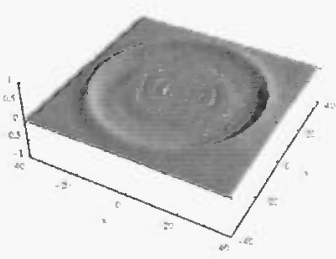

$t=30$

Figure 4. $\eta(x, y, t)$ at $t=0,5,15,30$, BBM-BBM, zero Neumann b. c.

that (1.1) is invariant under the transformation $x \mapsto-x, y \mapsto-y, u \mapsto-u, v \mapsto-v, \eta \mapsto \eta$. If we assume initial data invariant under the same transformation, then the uniqueness result for the initial-value problem implies that the solution satisfies for all $t$ the symmetric relations $u(x, y, t)=-u(-x,-y, t), v(x, y, t)=-v(-x,-y, t)$, $\eta(x, y, t)=\eta(-x,-y, t)$. Figure 2 shows one-dimensional cross sections of $\eta$ at $t=30$ in the $y, x$, and $y=x$ directions from the previous evolution; the dispersive nature of the oscillations is evident.

Figure 3 shows the evolution of the $\eta$ profile (BBM-BBM, zero Neumann boundary conditions) emanating from two separated Gaussians, specifically from the initial data $\eta_{0}(x, y)=\Phi(x-15, y)+\Phi(x+15, y), \Phi(x, y)=$ $0.2 \exp \left(-\left(x^{2}+y^{2}\right) / 5\right), u_{0}(x, y)=v_{0}(x, y)=0$. (Bilinear elements-RK2, square mesh, $h=0.5, \Delta t=0.05$.) In Figure 4 the same system is integrated with the same numerical method and mesh sizes, but with initial data given by $\eta_{0}(x, y)=\exp \left(-\left(x^{2} / 5+y^{2} / 25\right)\right), u_{0}(x, y)=v_{0}(x, y)=0$. The associated Figure 5 depicts cross sections of the $\eta$ profiles at $t=30$ in the $y, x$, and $y=x$ directions. Observe that this initial profile yields an expanding wave with an "inverted $\mathrm{N}$ " shaped front, with small-size oscillations remaining behind. 

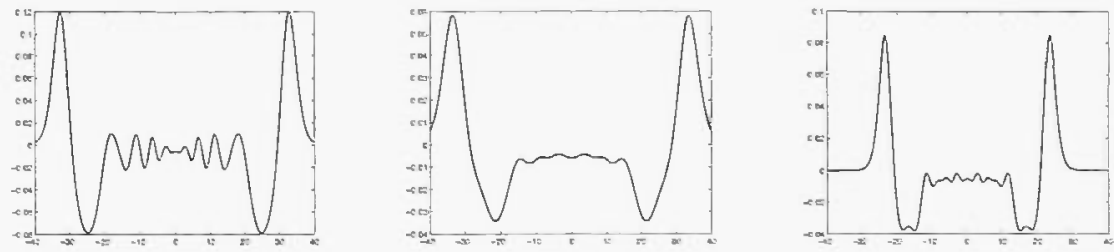

FIGURE 5. Cross sections in the $(0,1),(1,0)$ and $(1,1)$ directions of $\eta(x, y, t)$ at $t=30$. Evolution of Figure 4.

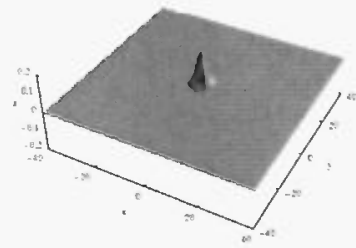

$t=0$

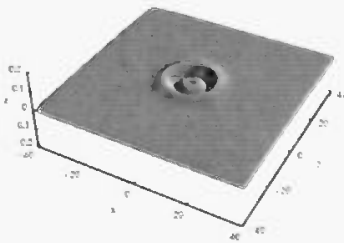

$t=10$

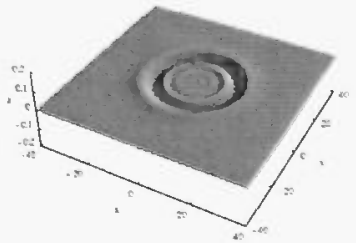

$t=20$

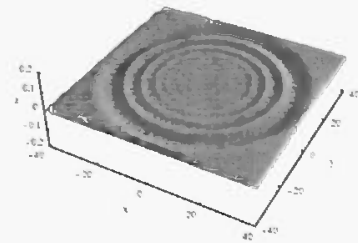

$t=40$

Figure 6. $\eta(x, y, t)$ at $t=0,10,20,40$, Bona-Smith, zero Dirichlet b. c.

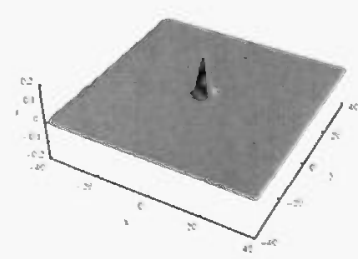

$t=0$

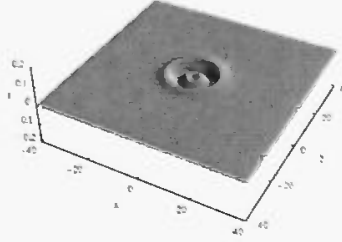

$t=10$

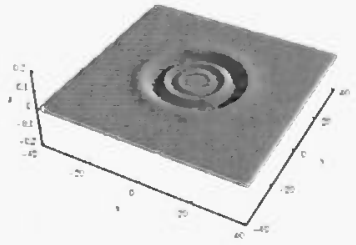

$t=20$

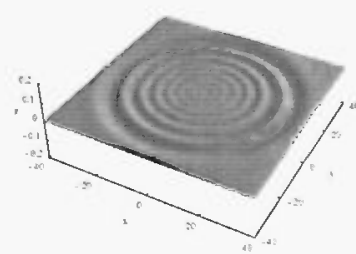

$t=40$

FIGURE 7. $\eta(x, y, t)$ at $t=0,10,20,40$, BBM-BBM, zero Dirichlet b. c.

In Figures 6-8 we present a comparison of the evolution of the $\eta$ component of the solution from radially symmetric initial data of the form $\eta_{0}(x, y)=0.2 \exp \left(-\left(x^{2}+y^{2}\right) / 5\right), u_{0}(x, y)=v_{0}(x, y)=0$, under the BBMBBM (Fig. 6) and the Bona-Smith system system (Fig. 7), both considered with zero Dirichlet boundary conditions (BBM-BBM: Bilinear elements-RK2, square mesh, $h=0.5, \Delta t=0.1$, Bona-Smith: Bicubic elementsRK4, square mesh, $h=\mathbf{1}, \Delta=0.2$.) Figure 8 shows the cross sections of the $\eta$ profiles of the two evolutions at $t=30$ in the directions $y, x$, and $y=x$. The speed and the amplitude of the outgoing front is approximately the same for both systems but the pattern of the oscillations behind the fronts are different: In the case of the Bona-Smith system the two outgoing wave trains have practically separated by $t=30$, while the larger in amplitude dispersive oscillatory tails of the BBM-BBM solution seem to be still interacting.

\subsection{Exact line solitary waves}

In the case of the one-dimensional BBM-BBM system an exact 'M-shaped' solitary wave is known to exist [10]. We present in Figure 9 the one-way 2D line analog: namely the $y$-independent propagation of $\eta$ in the positive 

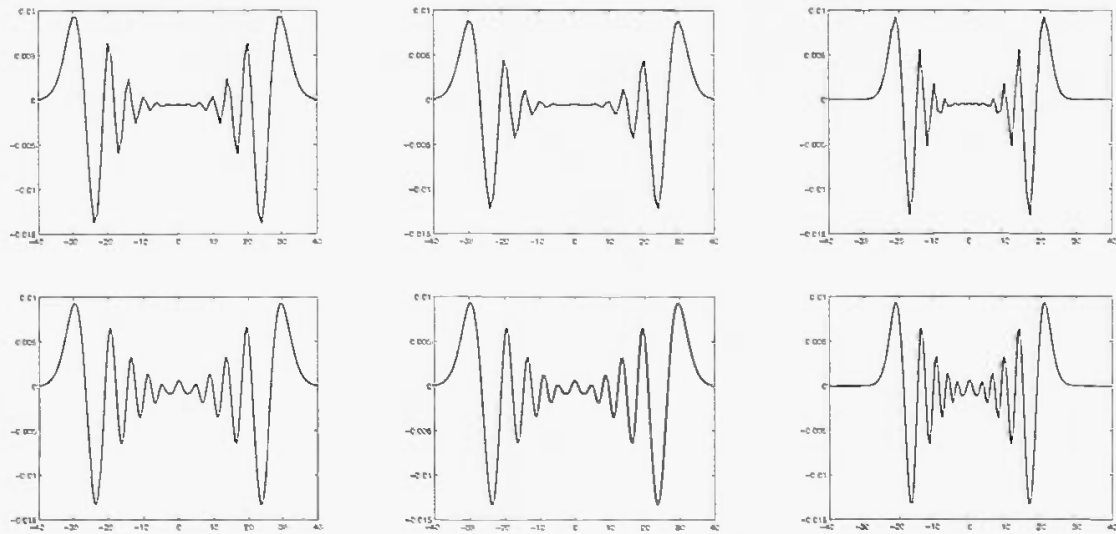

Figure 8. Cross sections in $(0,1),(1,0)$ and $(1,1)$ directions of $\eta(x, y, t)$ at $t=30$, Bona-Smith, (above) and BBM-BBM (below), zero Dirichlet b. c. Evolution of Figures 6 and 7.
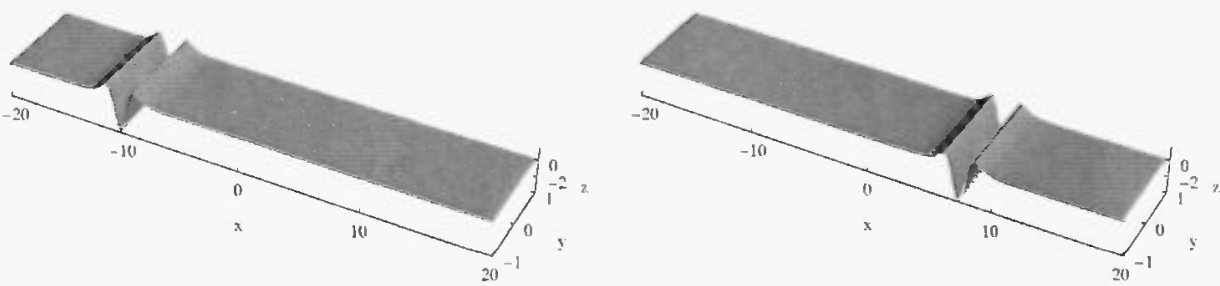

Figure 9. $\eta(x, y, t)$ at $t=0$ (left) and $t=7$ (right), BBM-BBM, zero Neumann b. c.

$x$-direction. This exact solution is given by the formulas

$$
\begin{aligned}
& \eta(x, y, t)=\frac{15}{4}\left(2 \operatorname{sech}^{2}\left(\frac{3}{\sqrt{10}}\left(x-\frac{5}{2} t\right)\right)-3 \operatorname{sech}^{4}\left(\frac{3}{\sqrt{10}}\left(x-\frac{5}{2} t\right)\right)\right), \\
& u(x, y, t)=\frac{15}{2} \operatorname{sech}^{2}\left(\frac{3}{\sqrt{10}}\left(x-\frac{5}{2} t\right)\right), \\
& v(x, y, t)=0 .
\end{aligned}
$$

BBM-BBM was integrated with zero Neumam boundary conditions on the rectangle $[-20,20] \times[-1,1]$. (Bilinear elements-RK2, square mesh, $h=0.1, \Delta t=0.01$.)

\subsection{Line solitary waves: Generation and resolution}

In Figures 10-16 we present numerical simulations of line solitary waves of the BBM-BBM system with zero Neumanm boundary conditions. Figure 10 shows the initial condition and the profile at $t=85$ of $\eta$; produced by the $x$-localized, $y$-independent initial data $\eta_{0}(x, y)=0.8 \exp \left(-x^{2} / 16\right), u_{0}(x, y)=v_{0}(x, y)=0$ on $[-70,70] \times[-5,5]$. (Bilinear elements-RK2, square mesh, $h=0.5, \Delta t=0.05$ ). Two $y$-independent wave trains emerge travelling in the positive and negative $x$ directions. The left-travelling wavetrain seems to be destroyed as it hits the $x=-70$ boundary leaving a small residue while the right-travelling one seems to have produced two line solitary waves plus a small dispersive tail by $t=85$.

More general initial conditions seem to be resolved into line solitary waves as $t$ grows. In Figure 11, the initial condition of $\eta$ was a small-amplitude sinusoidal perturbation in the $y$-direction of a line Gaussian in the 

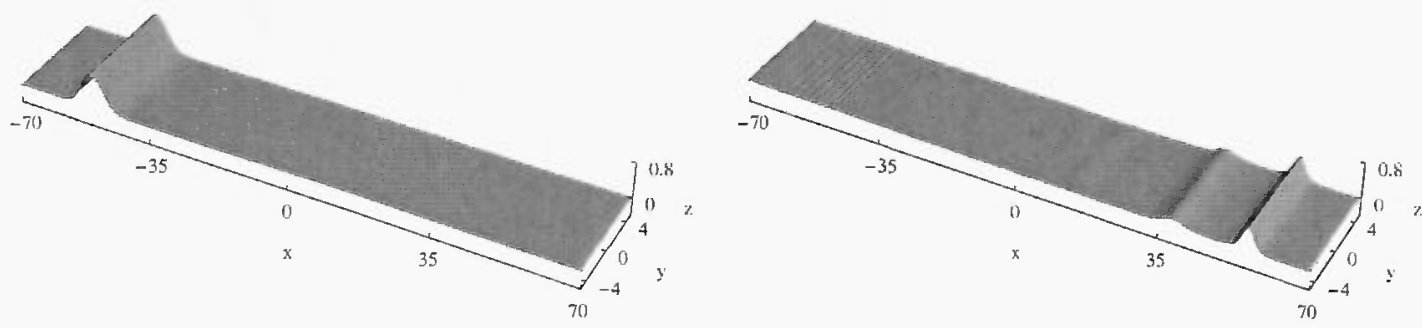

Figure 10. $\eta(x, y, t)$ at $t=0$ (left) and $t=85$ (right), BBM-BBM, zero Neumam b. c.
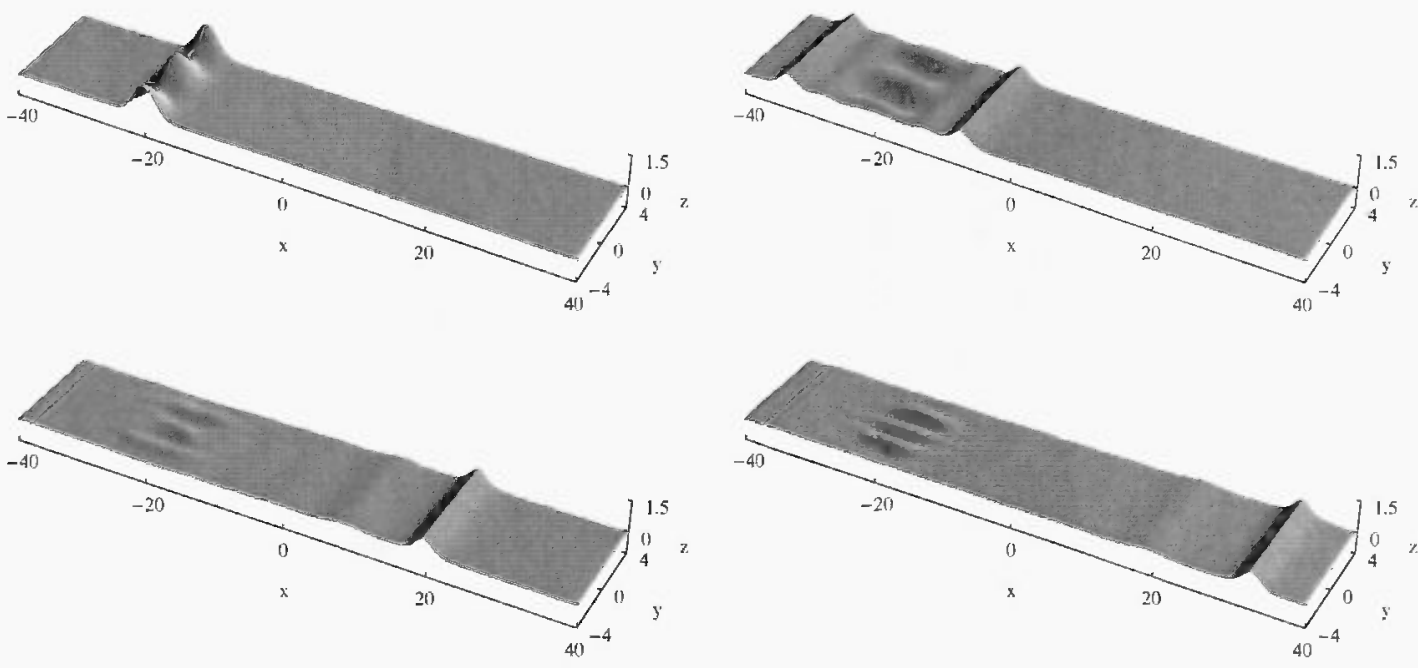

FIGURE 11. $\eta(x, y, t)$ at $t=0,10,30,40$. Initial profile $(6.1)$ with $\delta=0.5$, BBM-BBM, zero Neumann b. c.

$x$-direction. Specifically we took

$$
\eta_{0}(x, y)=\left(1+\delta \cos \left(\frac{\pi}{2} y\right)\right) \exp \left(-\frac{(x+20)^{2}}{5}\right), u_{0}(x, y)=v_{0}(x, y)=0
$$

in $[-40,40] \times[-4,4]$. Figure 11 shows the evolution of $\eta$ for $\delta=0.5$. (Bilinear elements-RK2, square mesh, $h=0.2, \Delta t=0.02$ ). The transverse perturbation decays and stays localized near $x=-20$; it does not seem to influence, as $t$, grows, the generation and propagation of line solitary waves in the $x$-direction.

It is worthwhile to note that if $y$-independent initial values $\eta_{0}, u_{0}, v_{0}$ are suitable chosen, then the solution that emerges moves mainly in one of the $x$-directions. In the case of the Bona-Smith family of systems (including the BBM-BBM case) the appropriate initial data for this purpose is $\eta_{0}(x, y)=\Phi(x), u_{0}(x, y)=\Phi(x)-\frac{1}{4} \Phi^{2}(x)$, $v_{0}(x, y)=0$, where $\Phi(x)=A \operatorname{sech}^{2}\left(\frac{1}{2} \sqrt{\frac{3 A}{c_{*}}} x\right), c_{s}=1+A / 2, c f$. e.g. [1]. The emerging $y$-independent solution moves in the positive $x$-direction shedding only a small amplitude left-travelling dispersive tail. In the example of Figure 12 we chose such initial data with $A=1$ on $[-100,100] \times[-2,2]$ and integrated both the BBM-BBM system and its symmetric analog (system (1.4) with $\varepsilon=1, a=c=0, b=d=1 / 6$ ). Both systems seem to produce nearly one-way travelling solutions; $c f$. Figure 12 for their $\eta$ profile at $t=40$, consisting of one main 

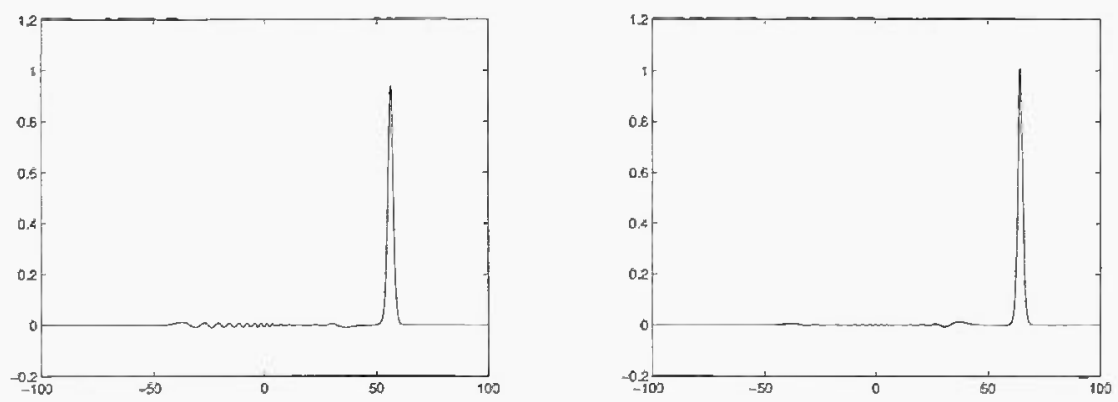

FIGURE 12. Comparison between BBM-BBM (left) and its symmetric version (right); $\eta(x, y, t)$ at $t=40$.
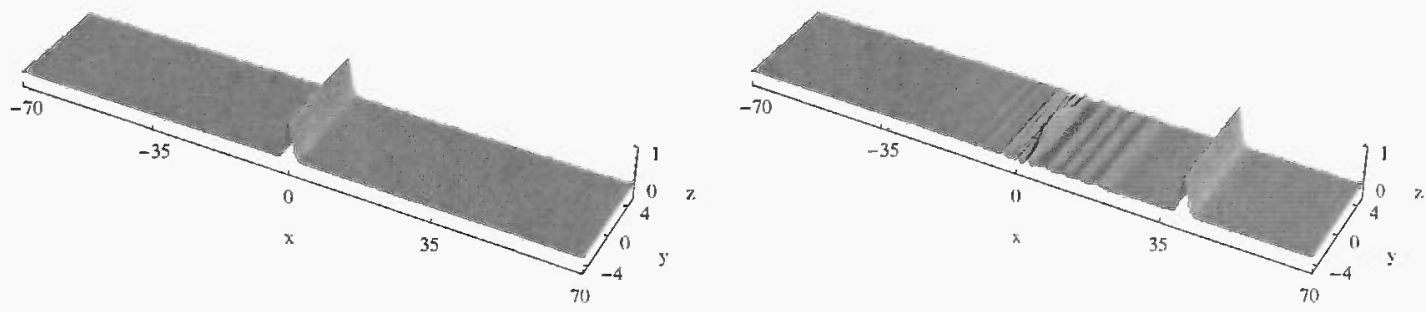

FIGURE 13. $\eta(x, y, t)$ at $t=0$ (left) and $t=40$ (right). Initial profile $(6.2)$ with $\delta=0.2$, BBM-BBM, zero Neumann b. c.

line solitary wave pulse. (Both systems were integrated with zero Neumann boundary conditions, using bilinear elements and RK2, square mesh $h=0.2, \Delta t=0.02$.) The symmetric BBM-BBM solitary wave is slightly higher and faster and its left-travelling residue is of smaller amplitude.

In Figures 13-18 we show the evolution of the $\eta$-component of the solution of the BBM-BBM system with such "one-way" initial data that was perturbed in the $y$-direction. Specifically we took on $[-70,70] \times[-4,4]$

$$
\begin{aligned}
& \eta_{0}(x, y)=\Phi(x, y) \\
& u_{0}(x, y)=\Phi(x, y)-\frac{1}{4} \Phi^{2}(x, y) \\
& v_{0}(x, y)=0 \\
& \Phi(x, y)=A \operatorname{sech}^{2}\left(\frac{1}{2} \sqrt{\frac{3 A}{c_{s}}} x+\delta \cos \left(\frac{\pi}{2} y\right)\right), c_{s}=1+\frac{A}{2}
\end{aligned}
$$

and integrated in time taking various values of $\delta$ and $A=1$. (Note that this perturbation is nonplyysical as it violates the irrotationality condition $v_{x}=u_{y}$ at $t=0$.) (Bilinear elements-RK2, square mesh, $h=0.2$, $\Delta t=0.02$.) In Figure $13(\delta=0.2)$ one right-travelling line solitary wave is produced. Larger values of $|\delta|$ give larger amplitude left- and right-travelling 'dispersive' tails and one right-travelling 'main' line solitary pulse. (Possibly two such solitary waves emerge when $\delta=-0.5$.) Taking $|\delta| \geq 1$ leads to blow-up of the solution, as the negative excursion of the oscillations become and stay less than $\eta=-1$.

Figure 17 shows the evolution of $\eta$ of the solution of the BBM-BBM system with initial data of the type (6.2) but now with $\Phi(x, y)=\left(1+\delta \cos \left(\frac{\pi}{2} y\right)\right) A \operatorname{sech}^{2}\left(\frac{1}{2} \sqrt{\frac{3 A}{c_{s}}}(x+20)\right), c_{s}=1+A / 2$. We took $A=1$ and $\delta=0.5$ 

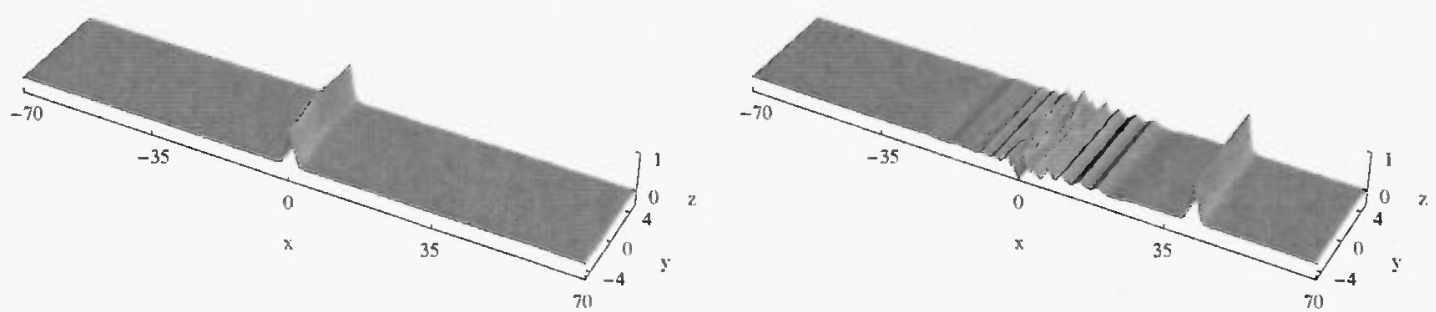

FIGURE 14. $\eta(x, y, t)$ at $t=0$ (left) and $t=40$ (right). Initial profile (6.2) with $\delta=0.5$, BBM-BBM, zero Neumann b. c.
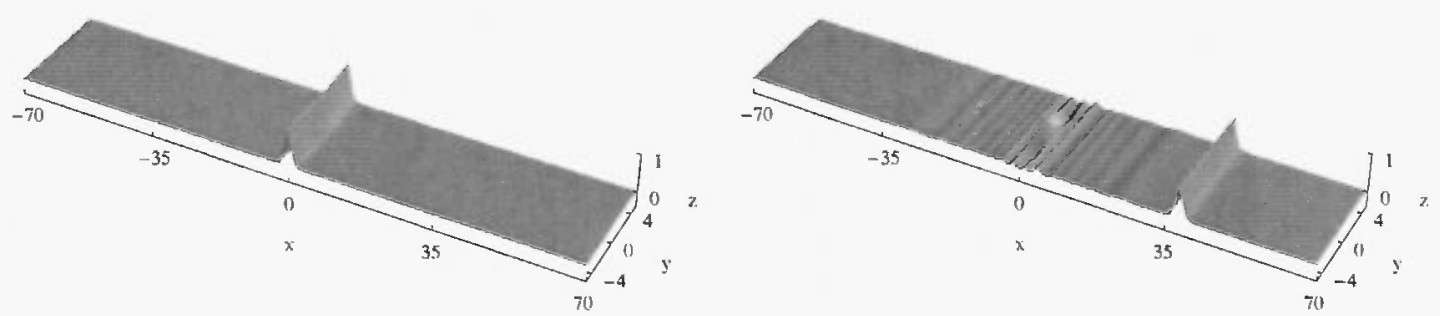

Figure 15. $\eta(x, y, t)$ at $t=0$ (left) and $t=40$ (right). Initial profile (6.2) with $\delta=-0.2$, BBM-BBM, zero Neumanu b. c.
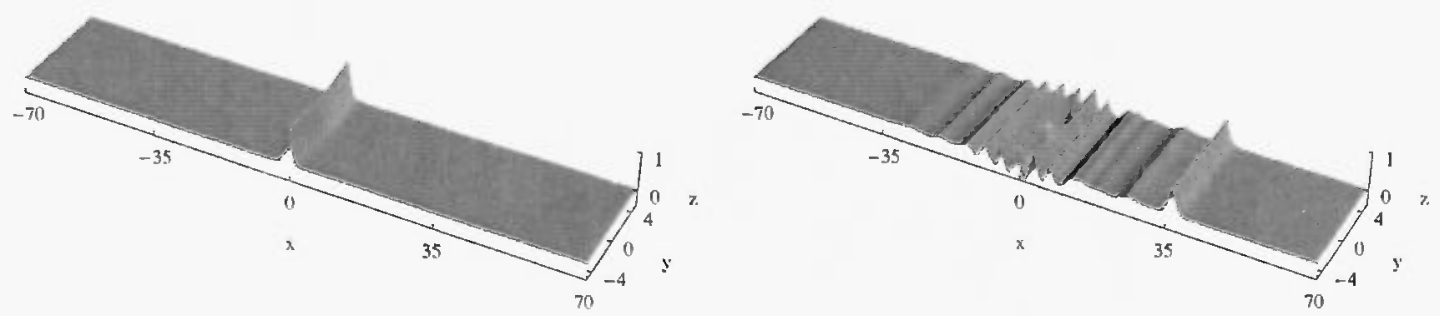

Figure 16. $\eta(x, y, t)$ at $t=0$ (left) and $t=40$ (right). Initial profile (6.2) with $\delta=-0.5$, BBM-BBM, zero Neumann b. c.

(bilinear elements-RK2, square mesh on $[-40,40] \times[-4,4], h=0.2, \Delta t=0.02$ ) and observed the generation of at least one solitary wave plus dispersive tail. A cross-section of the solution of Figure 17 appears in Figure 18.

\subsection{Collision of line solitary waves}

In Figure 19 we show the $\eta$-profile of the collision of two BBM-BBM line solitary waves travelling in opposite directions. (Bilinear elements-RK2, square mesh on $[-60,60] \times[-2,2], h=0.2, \Delta t=0.02$ ). As initial condition we used the data $\eta_{0}(x, y)=\Phi(x+30)+\Phi(x-3), u_{0}(x, y)=\Phi(x+30)+\frac{1}{4} \Phi^{2}(x+30)-\left(\Phi(x-30)+\frac{1}{4} \Phi^{2}(x-30)\right)$, where $\Phi(x)=\operatorname{sech}^{2}(x / \sqrt{2})$. Figure 20 shows cross-sections of the solution near the interaction location and time. The interaction features are as expected from one-dimensional simulations. 

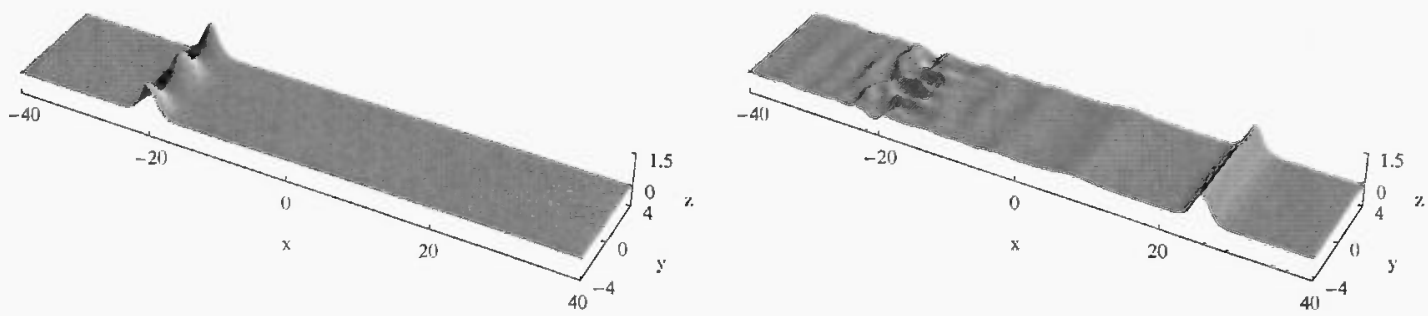

Figure 17. $\eta(x, y, t)$ at $t=0$ (left) and $t=30$ (right). Initial profile similar to (6.2), with $\delta=0.5, \mathrm{BBM}-\mathrm{BBM}$, zero Neumann b. c.
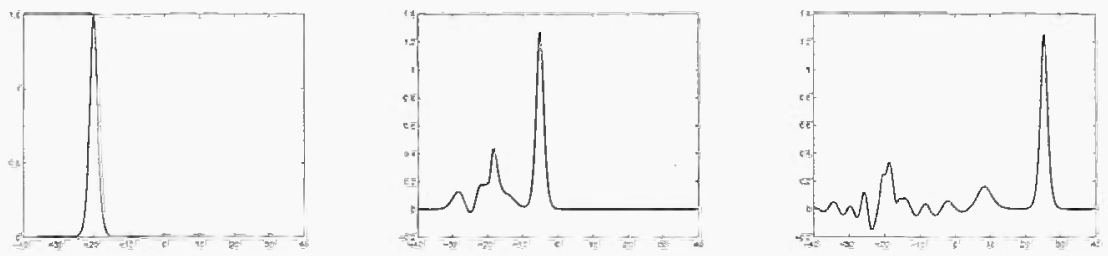

Figure 18. Cross sections of $\eta(x, y, t)$ at $t=0,10,30$, BBM-BBM, zero Neumann b. c. Evolution of Figure 17.

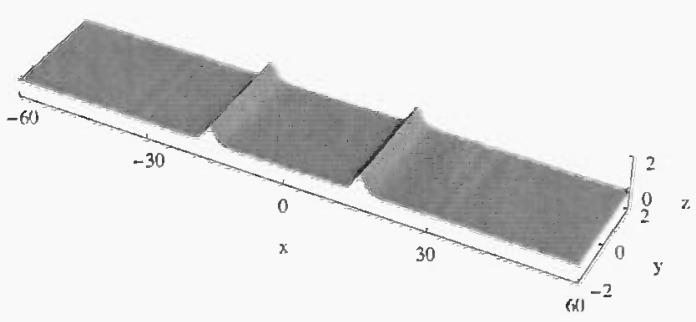

$t=10$

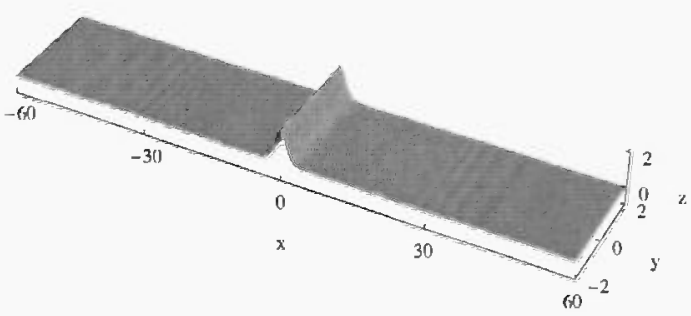

$t=22$

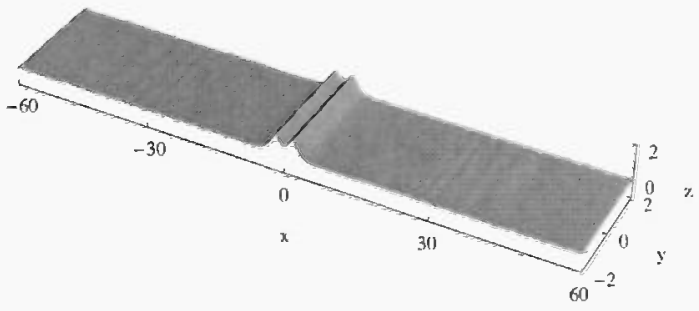

$t=20$

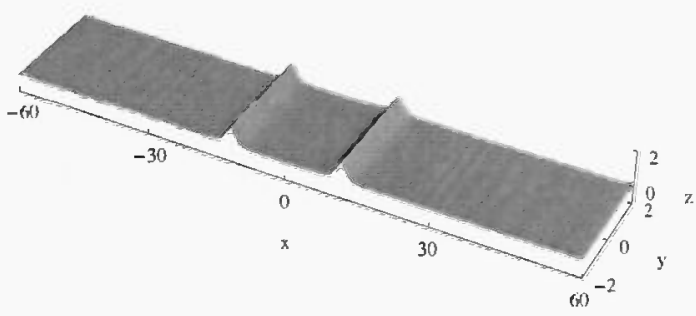

$t=30$

Figure 19. $\eta$ profile of the collision of two line solitary waves, BBM-BBM, zero Neumann b. $c$.

\subsection{Interaction of two line solitary waves travelling in orthogonal directions}

In Figure 21 we show the $\eta$-profile at $t=0$ and at $t=40$ of the solution of the BBM-BBM system on $[-40,40] \times[-40,40]$ with initial data $\eta_{0}(x, y)=\exp \left(-(x-25)^{2} / 5\right)+\exp \left(-(y-25)^{2} / 5\right), u_{0}(x, y)=v_{0}(x, y)=0$. 

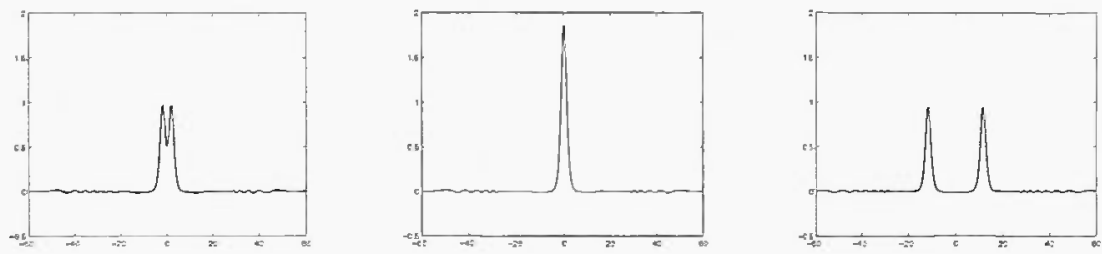

FIguRE 20. Cross sections along the $x$-axis of $\eta(x, y, t)$ at $t=20,22,35$, BBM-BBM, zero Neumamn b. c. Evolution of Figure 19.
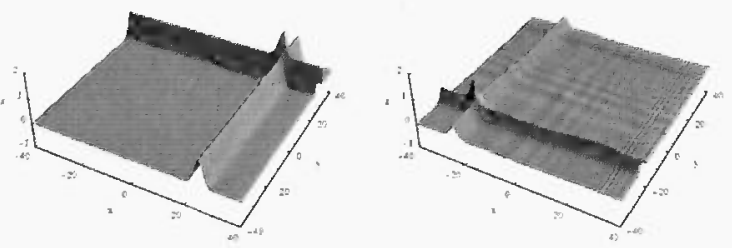

FIgURE 21. $\eta(x, y, t)$ for $t=0,40$, BBM-BBM, zero Neumamn b. c.
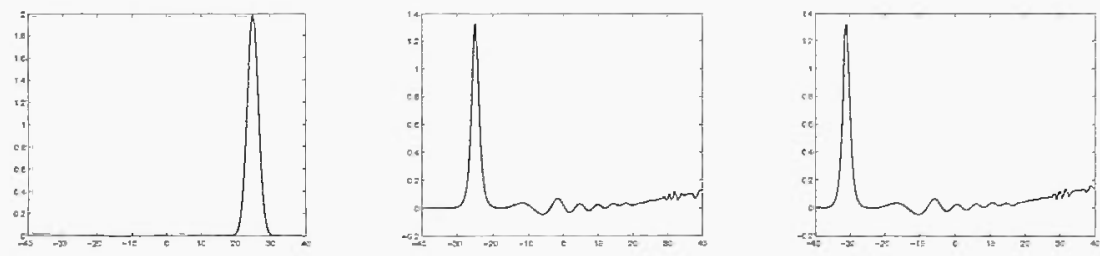

FIGURE 22. Cross sections along $(1,1)$ direction of $\eta(x, y, t)$ at $t=0,40,45$, BBM-BBM, zero Neumamn b. c. Evolution of Figure 21.

(Bilinear elements-RK2, square mesh, $h=0.5, \Delta t=0.05$.) Two line solitary waves are produced travelling in the negative $x$ - and $y$-directions respectively. Study of the details of their interaction reveals that it is inelastic and that there is a phase shift. Figure 22 shows the cross sections of $\eta$ in the $x=y$ direction at $t=0,40,45$.

\subsection{Reflection of line solitary waves at boundaries}

In Figure 23 we show the reflection of the $\eta$ component of a line solitary wave of the BBM-BBM system on $[-50,50] \times[-1,1]$. We used initial data of the form (6.2) with $A=0.1, \delta=0$, with $\Phi(x+20, y)$ instead of $\Phi(x, y)$. (Linear elements-RK2, regular triangular mesh with 9672 elements, $\Delta t=0.01$.) We used zero Neumann boundary conditions for $\eta$ on the whole boundary. The boundary conditions for $u$ and $v$ were homogeneous Dirichlet on $x=-50$ and $x=50$ and homogeneous Neumann on $y=-1$ and $y=1$. The solitary wave collides with the boundary and turns back, apparently retaining its amplitude, even after its interaction with the right-travelling dispersive tail of order of magnitude $10^{-3}$. 


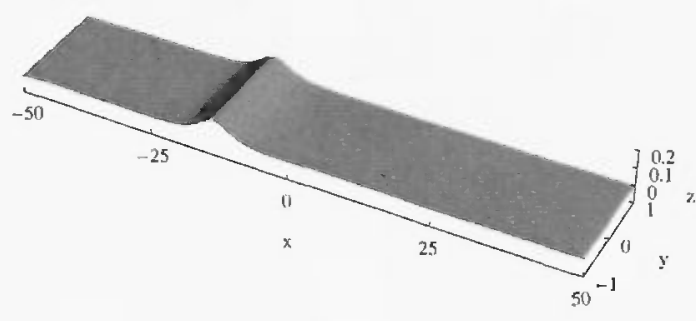

$t=10$

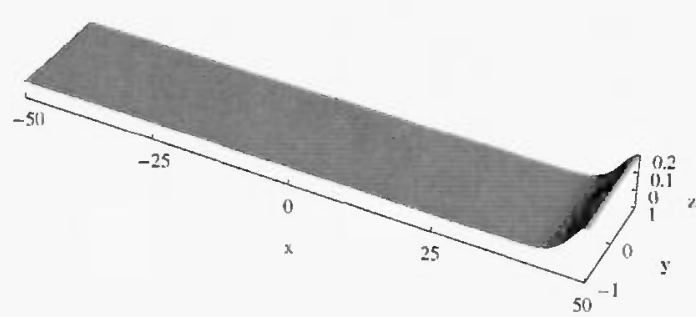

$t=100$

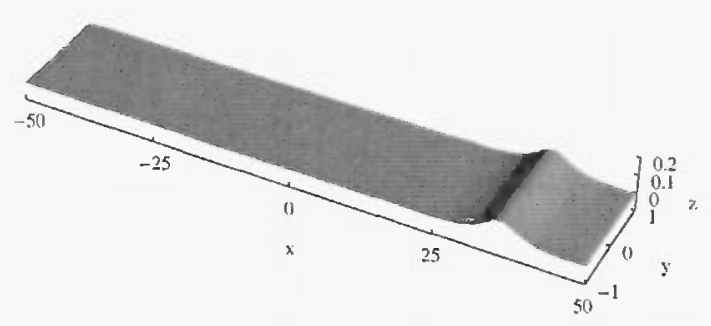

$t=120$

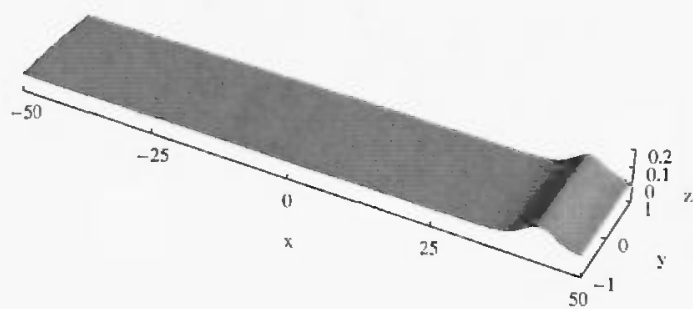

$t=90$

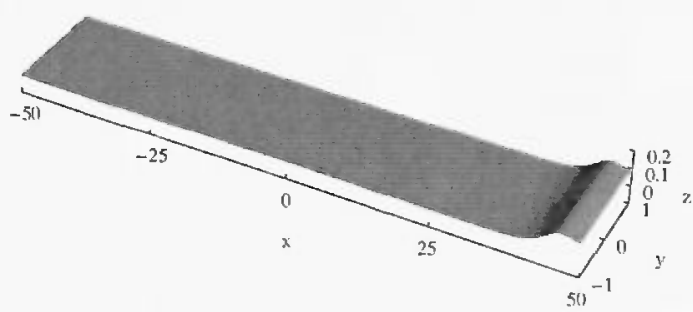

$\imath=105$

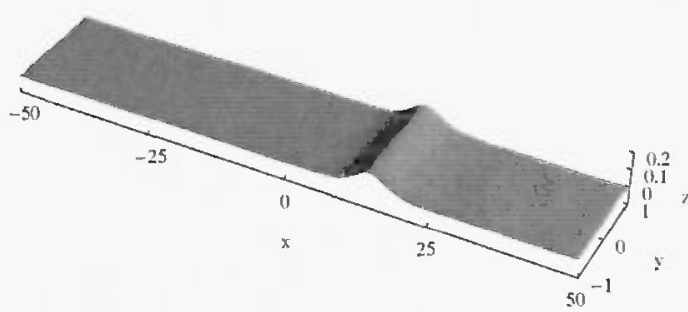

$t=150$

FIGURE 23. $\eta(x, y, t)$ for $t=10,90,100,105,120,150$, BBM-BBM, mixed b. c..

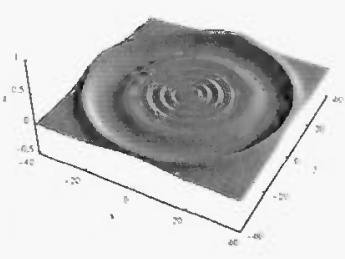

$t=35$

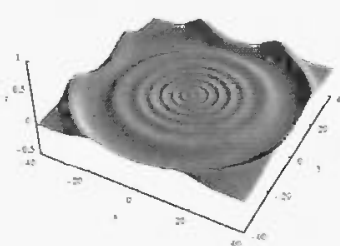

$t=40$

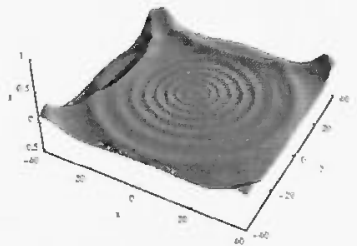

$t=50$

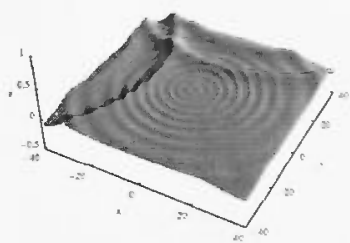

$t=60$

Figurls 24. $\eta(x, y, t)$ for $t=35,40,50,60$, BBM-BBM, mixed b. c.

\subsection{Reflection of expanding symmetric waves at boundaries}

In Figure 24 we show the reflection from one part of the boundary of an expanding symmetric wave of the BBM-BBM system. For this experiment we used as initial data the functions $\eta_{0}(x, y)=2 \exp \left(-\left(x^{2}+y^{2}\right) / 5\right)$, $u_{0}(x, y)=v_{0}(x, y)=0$. (Linear elements-RK2, regular triangular mesh with 84992 elements, $\Delta t=0.01$.) We used zero Neumam boundary conditions for $\eta$ on the whole boundary, zero Dirichlet data for $u$ and $v$ on 
$x=-40$ and $y=40$, and zero Neumann boundary data for $u$ and $v$ on $x=40$ and $y=-40$. The expanding waves are reflected from the $x=-40$ and $y=40$ parts of the boundary.

\section{REFERENCES}

[1] A.A. Alazman, J.P. Albert, J.L. Bonar M. Chen and J. Wu, Comparisons between the BBM equation and a Boussinesq system. Adv. Differential Equations 11 (2006) 121-166.

[2] D.C. Antonopoulos, The Boussinesq system of equations: Theory and numerical analysis. Ph.D. Thesis, University of Athens, 2000 (in Greek).

[3] D.C. Antonopoulos, V.A. Dougalis and D.E. Mitsotakis, Theory and numerical analysis of the Bona-Smith type systems of Boussinesq equations. (to appear).

[4] J.L. Bona and M. Chen, A Boussinesq system for two-way propagation of nonlinear dispersive waves. Physica $D$ 116 (1998) 191-224.

[5] J.L. Bona and R. Snith, A model for the two-way propagation of water waves in a channel. Moth. Proc. Camb. Phil. Soc. 79 (1976) $167-182$.

[6] J.L. Bona, M. Chen and J.-C. Saut, Boussinesq equations and other systems for small-amplitude long waves in nonlinear dispersive media: I. Derivation and Linear Theory. J. Nonlinear Sci. 12 (2002) 283-318.

[7] J.L. Bona, M. Chen and J.-C. Saut, Boussinesq equations and other systems for small-amplitude long waves in nonlinear dispersive media: II. The nonlinear theory. Nonlinearity 17 (2004) 925-952.

[8] J.L. Bona, T. Colin and D. Lannes, Long wave approximations for water waves. Arch. Rational Mech. Anal. 178 (2005) 373-410.

[9] S.C. Brenner and L.R. Scott, The Mathematical Theory of Finite Element Methods. Springer-Verlag; New York (1994).

[10] M. Chen, Exact traveling-wave solutions to bi-directional wave equations. Int. J. Theor. Phys. 37 (1998) $1547-1567$.

[11] M. Chen, Solitary-wave and multi pulsed traveling-wave solutions of Boussinesq systems. Applic. Analysis 75 (2000) $213-240$.

[12] V.A. Dougalis and D.E. Mitsotakis, Solitary waves of the Bona-Smith system, in Advances in scattering theory and biomedical engineering, D. Fotiadis and C. Massalas Eds., World Scientific, New Jersey (2004) 286-294.

[13] V.A. Dougalis, D.E. Mitsotakis and J.-C. Saut, On initial-boundary value problems for some Boussinesq systems in two space dimensions. (to appear).

[14] P. Grisvard, Quelques proprietés des espaces de Sobolev, utiles dans l'étude des équations de Navier-Stokes (1). Problèmes d'évolution, non linéaires, Séminaire de Nice (1974-1976).

[15] D.R. Kincaid, J.R. Respess, D.M. Young and R.G. Grimes, ITPACK 2C: A Fortran package for solving large sparse linear systems by adaptive accelerated iterative methods. ACM Trans. Math. Software 8 (1982) 302-322.

[16] R. Rannacher and R. Scott, Some optimal error estimates for piecewise linear finite element approximations. Math. Comp. 38 (1982) 437-445.

[17] A.H. Schatz and L.B. Wahlbin, On the quasj-optimality in $L_{\infty}$ of the $H_{1}^{\circ}$-projection into finite elements spaces. Math. Comp. 38 (1982) 1-22.

[18] M.H. Schultz, $L^{\infty}$ Multivariate approximation theory. SIAM J. Numer. Anal. 6 (1969) 161-183.

[19] M.H. Schultz, Approximation theory of multivatiate spline functions in Sobolev spaces. SIAM J. Numer. Anal. 6 (1969) 570-582.

[20] J.F. Toland, Existence of symmetric homoclinic orbits for systems of Euler-Lagrange equations. A.M.S. Proc. Symposia in Pure Mathematics 45 (1986) 447-459.

[21] G.B. Whitham, Linear and Non-linear Waves. Wiley, New York (1974). 